Proceedings of the Edinburgh Mathematical Society (2004) 47, 709-752 (C)

DOI:10.1017/S0013091503000907 Printed in the United Kingdom

\title{
SPACES OF LIPSCHITZ TYPE ON METRIC SPACES AND THEIR APPLICATIONS
}

\author{
DACHUN YANG ${ }^{1}$ AND YONG LIN ${ }^{2}$ \\ ${ }^{1}$ Department of Mathematics, Beijing Normal University, Beijing 100875, \\ People's Republic of China (dcyang@bnu.edu.cn) \\ ${ }^{2}$ Department of Mathematics, Information School, \\ Renmin (People) University of China, Beijing 100872, \\ People's Republic of China (yonglin@95777.com)
}

(Received 23 October 2003)

\begin{abstract}
New spaces of Lipschitz type on metric-measure spaces are introduced and they are shown to be just the well-known Besov spaces or Triebel-Lizorkin spaces when the smooth index is less than 1. These theorems also hold in the setting of spaces of homogeneous type, which include Euclidean spaces, Riemannian manifolds and some self-similar fractals. Moreover, the relationships amongst these Lipschitz-type spaces, Hajłasz-Sobolev spaces, Korevaar-Schoen-Sobolev spaces, Newtonian Sobolev space and Cheeger-Sobolev spaces on metric-measure spaces are clarified, showing that they are the same space with equivalence of norms. Furthermore, a Sobolev embedding theorem, namely that the Lipschitz-type spaces with large orders of smoothness can be embedded in Lipschitz spaces, is proved. For metric-measure spaces with heat kernels, a Hardy-Littlewood-Sobolev theorem is establish, and hence it is proved that Lipschitz-type spaces with small orders of smoothness can be embedded in Lebesgue spaces.
\end{abstract}

Keywords: space of homogeneous type; Lipschitz space; Besov space; Triebel-Lizorkin space; heat kernel; embedding theorem

2000 Mathematics subject classification: Primary 42B35

Secondary 46E35; 58J35; 43A99

\section{Introduction}

In $[\mathbf{2 5}]$ and $[\mathbf{2 4}]$ (see also [23]), Han, Lu and Yang introduced Besov spaces on spaces of homogeneous type. It was observed by Triebel in $[\mathbf{4 8}, \mathrm{pp} .159,160]$ that $d$-sets in $\mathbb{R}^{n}$ are spaces of homogeneous type and, furthermore, he asked there if Besov spaces on $d$-sets introduced by him in $[\mathbf{4 7}, \mathbf{4 8}]$, equivalently using either traces or quarkonial decompositions, are the same as those defined by Han, Lu and Yang in $[\mathbf{2 4 , 2 5}$. This was answered in the affirmative by Yang in [52]. In [47], Triebel also proved that $d$ sets in $\mathbb{R}^{n}$ include some self-similar fractals and, in particular, the post critically finite self-similar fractals that were introduced in $[\mathbf{3 3}, \mathbf{4 3}]$. Recently, Strichartz $[\mathbf{4 3}]$ introduced Hölder-Zygmund, Besov and Sobolev spaces on a class of post critically finite self-similar fractals and proved that for small orders of smoothness, these function spaces coincide 
with the function spaces defined by Jonsson and Wallin in [31]. Moreover, Strichartz further developed the theory including a proof of a version of the Sobolev embedding theorems.

In this paper, motivated by $[\mathbf{1 8}, \mathbf{3 0}-\mathbf{3 2}]$, we introduce some new spaces of Lipschitz type on metric-measure spaces and show that these new Lipschitz-type spaces are just the well-known Besov spaces or Triebel-Lizorkin spaces introduced in [23-26] when the smooth index is less than 1. Our results also indicate that some of the Hölder-Zygmund spaces of Strichartz [43] are the same as some of our function spaces for small orders of smoothness (see also $[\mathbf{3 1}, \mathbf{4 8}, \mathbf{5 2}]$ ). We further develop the theory of these function spaces in the setting of spaces of homogeneous type. This class includes $\mathbb{R}^{n}$, some Riemannian manifolds, some self-similar fractals and, in particular, the post critically finite self-similar fractals of $[\mathbf{3 3}, \mathbf{4 3}]$. Thus, as well as indicating how to define Lipschitz-type spaces of order no less than 1 related to Besov and Triebel-Lizorkin spaces on spaces of homogeneous type (particularly on metric-measure spaces and fractals), our results also give some new characterizations of Besov and Triebel-Lizorkin spaces of order less than 1 using discrete square fractional derivatives (see $[\mathbf{1 3}, \mathbf{1 4}, \mathbf{2 3}]$ ). Moreover, Theorem 3.12 below contains both a discrete and an inhomogeneous version of Theorem 2 of $[\mathbf{1 3}]$. We further clarify the relationships amongst our Lipschitz-type spaces, Hajłasz-Sobolev spaces $[\mathbf{1 9}, \mathbf{2 0}, \mathbf{2 8}$, Korevaar-Schoen-Sobolev spaces $[\mathbf{2 9}, \mathbf{3 4}]$, Newtonian Sobolev spaces [40] and CheegerSobolev spaces [4] on metric-measure spaces, based on the related results of $[\mathbf{2 9}, \mathbf{3 5}]$. In fact, we show that they coincide with equivalence of norms (see Corollary 4.5 below). Furthermore, we establish versions of the Sobolev embedding theorems. To be precise, we prove that our Lipschitz-type spaces with large orders of smoothness can be embedded in Lipschitz spaces (see Proposition 2.5 and Corollary 2.6 below); some of our results for $p=2$ were obtained in $[\mathbf{1 6}, \mathbf{1 8}]$. Finally, motivated by $[\mathbf{1 8}]$ (see also $[\mathbf{1 6}, \mathbf{4 3}]$ ), we consider metric-measure spaces with a heat kernel satisfying certain estimates (see (5.7) and (5.18) below), which are known to be satisfied by heat kernels on many fractals, manifolds, graphs and groups (see $[\mathbf{3}, \mathbf{5}, \mathbf{1 1}, \mathbf{1 5}, \mathbf{1 7}, \mathbf{4 3}, \mathbf{5 0}, \mathbf{5 1}]$ ). By establishing HardyLittlewood-Sobolev theorems on such metric-measure spaces (see Theorem 5.4 below), we can prove Sobolev embedding theorems for our Lipschitz-type spaces with small orders of smoothness, that is, that they can be embedded in Lebesgue spaces (see Theorem 5.6 below).

As mentioned above, Strichartz [43] introduced Sobolev and Besov spaces on a class of post critically finite self-similar fractals. Observing that post critically finite self-similar fractals are spaces of homogeneous type (see (1.2) and (1.3) in [43]), it would be of interest to clarify if there exists any connection between the Sobolev and Besov spaces of Strichartz and our Lipschitz-type spaces, which is still as yet unknown.

Let us now recall some definitions and notation on spaces of homogeneous type. A quasi-metric $\rho$ on a set $X$ is a function $\rho: X \times X \rightarrow[0, \infty)$ satisfying

(i) $\rho(x, y)=0$ if and only if $x=y$;

(ii) $\rho(x, y)=\rho(y, x)$ for all $x, y \in X$; 
(iii) there exists a constant $A \in[1, \infty)$ such that for all $x, y$ and $z \in X$,

$$
\rho(x, y) \leqslant A[\rho(x, z)+\rho(z, y)] .
$$

Any quasi-metric defines a topology for which the balls

$$
B(x, r)=\{y \in X: \rho(y, x)<r\}
$$

for all $x \in X$ and all $r>0$ form a basis.

Throughout, we set $\operatorname{diam} X=\sup \{\rho(x, y): x, y \in X\}$. We also adopt the following conventions. By $f \sim g$, we mean that there is a constant $C>0$, independent of the main parameters, such that $C^{-1} g<f<C g$. We denote by $C$ a positive constant which is independent of the main parameters and which may vary from line to line. Constants with subscripts, such as $C_{1}$, do not change from one occurrence to another. We denote $\mathbb{N} \cup\{0\}$ by $\mathbb{Z}_{+}$and, for any $q \in[1, \infty]$, we denote by $q^{\prime}$ the conjugate index satisfying $1 / q+1 / q^{\prime}=1$. For any set $A$, we denote by $\chi_{A}$ the characteristic function of $A$. If $X_{1}$ and $X_{2}$ are two quasi-Banach spaces, $B_{1} \subset B_{2}$ means that there is a constant $C>0$ such that, for all $f \in B_{1}$,

$$
\|f\|_{B_{2}} \leqslant C\|f\|_{B_{1}} .
$$

Definition 1.1 (see [23]). Let $d>0$ and $0<\theta \leqslant 1$. A space of homogeneous type, $(X, \rho, \mu)_{d, \theta}$, is a set $X$ together with a quasi-metric $\rho$ and a non-negative Borel regular measure $\mu$ on $X$ such that there exists a constant $C_{0}>0$ satisfying the condition that for all $0<r<\operatorname{diam} X$ and for all $x, x^{\prime}, y \in X$,

$$
\mu(B(x, r)) \sim r^{d}
$$

and

$$
\left|\rho(x, y)-\rho\left(x^{\prime}, y\right)\right| \leqslant C_{0} \rho\left(x, x^{\prime}\right)^{\theta}\left[\rho(x, y)+\rho\left(x^{\prime}, y\right)\right]^{1-\theta} .
$$

Obviously, $d$ can be regarded as the Hausdorff dimension of $X$ (see [37]). Moreover, if $\rho$ is a metric, then $\theta$ in (1.2) can be taken to be 1 . If $X=\mathbb{R}^{n}, \rho$ is the usual Euclidean metric and $\mu$ is the $n$-dimensional Lebesgue measure, then $d=n$ and $\theta=1$.

The notion of space of homogeneous type defined above is a variant of the spaces of homogeneous type introduced by Coifman and Weiss in [7]. Macías and Segovia [36] proved that one can replace the quasi-metric $\rho$ of a homogeneous-type space in the sense of Coifman and Weiss by another quasi-metric $\bar{\rho}$, which yields the same topology on $X$ as $\rho$, and such that $(X, \bar{\rho}, \mu)$ is a space satisfying Definition 1.1 with $d=1$.

Spaces of homogeneous type include Euclidean space, the $n$-torus in $\mathbb{R}^{n}, C^{\infty}$-compact Riemannian manifolds, boundaries of Lipschitz domains and, in particular, the Lipschitz manifolds of Triebel [49] and isotropic and anisotropic $d$-sets in $\mathbb{R}^{n}$. It was proved by Triebel in [47] that the isotropic and anisotropic $d$-sets in $\mathbb{R}^{n}$ include various kinds of self-affine fractals, for example, the Cantor set (see also [37]), the generalized Sierpinski carpet, the fern-like fractals, Picasso-Xmas tree fractals and Oval-Ferny fractals (see [48], $[\mathbf{2}],[\mathbf{3}]$ and $[\mathbf{1 8}])$. 
The organization of the paper is as follows. In the next section, $\S 2$, motivated by Jonsson [30] (see also [18]), we introduce some new Lipschitz-type spaces on spaces of homogeneous type and establish some basic properties of these spaces, including the Sobolev embedding theorems. In particular, we show that there is a self-similar fractal in $\mathbb{R}^{2}$ with Hausdorff dimension $d>1$ and a Lipschitz function defined on it with exponent $d$. Thus, unlike the Euclidean case, Lipschitz spaces of order greater than 1 may be non-trivial on fractals or metric-measure spaces. This fact is well known (see $[\mathbf{3 0}, \mathbf{3 2}, \mathbf{3 9}]$ ) and one simple example in the one-dimensional case is provided in [30] by considering $X$ consisting of two disjoint intervals in $\mathbb{R}^{n}$. In $\S 3$, we discuss the relationships between our Lipschitz-type spaces and Besov and Triebel-Lizorkin spaces on spaces of homogeneous type. Section 4 is devoted to the study of the relationships amongst Lipschitz-type spaces, Hajłasz-Sobolev spaces and Korevaar-Schoen-Sobolev spaces on Ahlfors $d$-regular measure spaces. Finally, in $\S 5$, we study metric spaces with a heat kernel and we establish Sobolev embedding theorems into Lebesgue spaces of these Lipschitz-type spaces with small orders of smoothness.

\section{Spaces of Lipschitz type}

Let us first recall the well-known definition of the usual Lipschitz space (see also [30]). Let $X$ be a space of homogeneous type, $s>0$ and $C_{1}>0$. A function $f \in L^{\infty}(X)$ belongs to $L(s, X)$ if

$$
\|f\|_{\dot{L}(s, X)}=\operatorname{cosup}_{\substack{\mu, y \in X \\ 0<\rho(x, y)<C_{1}}} \frac{|f(x)-f(y)|}{\rho(x, y)^{s}}<\infty
$$

and we define

$$
\|f\|_{L(s, X)}=\max \left(\|f\|_{L^{\infty}(X)},\|f\|_{\dot{L}(s, X)}\right) .
$$

Obviously, the Lipschitz space $L(s, X)$ is independent of the choice of $C_{1}$.

The Lipschitz-type spaces, $L(s, p, q, X)$ defined below, are versions, on spaces of homogeneous type, of the Lipschitz spaces on $d$-sets of $\mathbb{R}^{n}$ in $[\mathbf{3 0}]$ and $[\mathbf{3 2}]$ (see also $[\mathbf{1 6}, \mathbf{1 8}]$ for the case of $p=2$ and $q=\infty)$. The Lipschitz-type spaces, $L_{\mathrm{b}}(s, p, q, X)$, are new.

Definition 2.1. Let $0<p, q \leqslant \infty$, let $s>0$ and let $C_{2}>0$ be a positive constant.

(i) A real-valued function $f$ defined $\mu$-almost everywhere on $X$ belongs to the Lipschitz-type space $L(s, p, q, X)$ if $f \in L^{p}(X)$ and

$$
\begin{aligned}
\|f\|_{\dot{L}(s, p, q, X)} & =\left\{\sum_{\nu=0}^{\infty} 2^{\nu s q}\left(2^{\nu d} \int_{X} \int_{B\left(x, C_{2} 2^{-\nu}\right)}|f(x)-f(y)|^{p} \mathrm{~d} \mu(y) \mathrm{d} \mu(x)\right)^{q / p}\right\}^{1 / q} \\
& <\infty .
\end{aligned}
$$

Moreover, we define

$$
\|f\|_{L(s, p, q, X)}=\|f\|_{L^{p}(X)}+\|f\|_{\dot{L}(s, p, q, X)} .
$$


(ii) A real-valued function $f$ defined $\mu$-almost everywhere on $X$ belongs to the Lipschitz-type space $L_{\mathrm{b}}(s, p, q, X)$ if $f \in L^{p}(X)$ and

$$
\begin{aligned}
\|f\|_{\dot{L}_{\mathrm{b}}(s, p, q, X)} & =\left\{\sum_{\nu=0}^{\infty} 2^{\nu s q}\left(\int_{X}\left[2^{\nu d} \int_{B\left(x, C_{2} 2^{-\nu}\right)}|f(x)-f(y)| \mathrm{d} \mu(y)\right]^{p} \mathrm{~d} \mu(x)\right)^{q / p}\right\}^{1 / q} \\
& <\infty
\end{aligned}
$$

Moreover, we define

$$
\|f\|_{L_{\mathrm{b}}(s, p, q, X)}=\|f\|_{L^{p}(X)}+\|f\|_{\dot{L}_{\mathrm{b}}(s, p, q, X)} .
$$

It is easy to see that the spaces $L(s, p, q, X)$ and $L_{\mathrm{b}}(s, p, q, X)$ are independent of the choice of $C_{2}>0$ (see also [30]). We remark that the space $L(s, p, q, X)$ is just a variant, on the homogeneous-type space, of the $\operatorname{space} \operatorname{Lip}(s, p, q, F)[\mathbf{3 0}]$, where $F$ is a $d$-set in $\mathbb{R}^{n}$. Moreover, as pointed out in [30], for $s>0$, we have

$$
L(s, \infty, \infty, X)=L(s, X) .
$$

If $X=\mathbb{R}^{n}$, then the continuous versions of $\|\cdot\|_{L(s, p, q, X)}$ and $\|\cdot\|_{L_{\mathrm{b}}(s, p, q, X)}$ are known to be equivalent to quasi-norms of Besov spaces $B_{p q}^{s}\left(\mathbb{R}^{n}\right)$ if $0<s<1,1 \leqslant p \leqslant \infty$ and $0<q \leqslant \infty$ (see Theorem 3.5.1 in [45, p. 187]). One of the main aims of this paper is to prove, in Theorem 3.8 below, that this is also true on spaces of homogeneous type.

Let us first establish some basic properties of $L(s, p, q, X)$ and $L_{\mathrm{b}}(s, p, q, X)$.

\section{Proposition 2.2.}

(i) If $s>0$ and $0<p, q \leqslant \infty$, then the spaces $L(s, p, q, X)$ and $L_{\mathrm{b}}(s, p, q, X)$ are quasi-Banach spaces.

(ii) If $s>0,1 \leqslant p \leqslant \infty$ and $0<q \leqslant \infty$, then

$$
L(s, p, q, X) \subset L_{\mathrm{b}}(s, p, q, X) .
$$

(iii) If $0<q_{0} \leqslant q_{1} \leqslant \infty, 0<p \leqslant \infty$ and $s>0$, then

$$
L\left(s, p, q_{0}, X\right) \subset L\left(s, p, q_{1}, X\right) \quad \text { and } \quad L_{\mathrm{b}}\left(s, p, q_{0}, X\right) \subset L_{\mathrm{b}}\left(s, p, q_{1}, X\right) .
$$

(iv) If $0<q_{0}, q_{1} \leqslant \infty, 0<p \leqslant \infty, s>0$ and $\epsilon>0$, then

$$
L\left(s+\epsilon, p, q_{0}, X\right) \subset L\left(s, p, q_{1}, X\right) \quad \text { and } \quad L_{\mathrm{b}}\left(s+\epsilon, p, q_{0}, X\right) \subset L_{\mathrm{b}}\left(s, p, q_{1}, X\right) .
$$

Proof. We first verify (i). Obviously, we need only verify the completeness of the spaces $L(s, p, q, X)$ and $L_{\mathrm{b}}(s, p, q, X)$. We prove this only for the space $L(s, p, q, X)$. The details of the proof for the space $L_{\mathrm{b}}(s, p, q, X)$ are left to the reader. Let $\left\{f_{j}\right\}_{j=1}^{\infty}$ be a Cauchy sequence in $L(s, p, q, X)$. Then it is also a Cauchy sequence in $L^{p}(X)$ since $L(s, p, q, X) \subset L^{p}(X)$. By the completeness of $L^{p}(X)$, it is easy to see that there exists $f$ 
in $L^{p}(X)$ such that $\lim _{j \rightarrow \infty}\left\|f_{j}-f\right\|_{L^{p}(X)}=0$ and $\lim _{j \rightarrow \infty} f_{j}(x)=f(x)$ a.e. on $X$. For any $\epsilon>0$, there exists an $N \in \mathbb{N}$ such that if $k, l>N$,

$$
\left\|f_{k}-f_{l}\right\|_{L(s, p, q, X)}<\epsilon .
$$

By Fatou's lemma, it follows that

$$
\begin{aligned}
\epsilon> & \lim _{k \rightarrow \infty}\left\|f_{k}-f_{l}\right\|_{L(s, p, q, X)} \\
= & \lim _{k \rightarrow \infty}\left\|f_{k}-f_{l}\right\|_{L^{p}(X)} \\
& +\lim _{k \rightarrow \infty}\left\{\sum _ { \nu = 0 } ^ { \infty } 2 ^ { \nu s q } \left[2^{\nu d} \int_{X} \int_{B\left(x, C_{2} 2^{-\nu}\right)} \mid f_{k}(x)-f_{l}(x)\right.\right. \\
\geqslant & \left.\left.\left\|f_{l}-f\right\|_{L^{p}(X)}(y)+\left.f_{l}(y)\right|^{p} \mathrm{~d} \mu(y) \mathrm{d} \mu(x)\right]^{q / p}\right\}^{1 / q} \\
& +\left\{\sum_{\nu=0}^{\infty} 2^{\nu s q}\left[2^{\nu d} \int_{X} \int_{B\left(x, C_{2} 2^{-\nu}\right)}\left|f_{l}(x)-f(x)-f_{l}(y)+f(y)\right|^{p} \mathrm{~d} \mu(y) \mathrm{d} \mu(x)\right]^{q / p}\right\}^{1 / q} \\
= & \left\|f_{l}-f\right\|_{L(s, p, q, X)} .
\end{aligned}
$$

From this and the fact that $f_{l} \in L(s, p, q, X)$, it is easy to deduce that $f \in L(s, p, q, X)$ and hence $f_{l} \rightarrow f$ in $L(s, p, q, X)$ as $l \rightarrow \infty$. Thus, the space $L(s, p, q, X)$ is a quasi-Banach space.

(ii) can be easily deduced from the Hölder inequality.

The proofs of (iii) and (iv) are similar to those of Proposition 2 in [44, p. 47]. We omit the details here.

This completes the proof of Proposition 2.2.

We now introduce new function spaces, $L_{\mathrm{t}}(s, p, q, X)$, on spaces of homogeneous type, which are variations of Lipschitz-type spaces, $L_{\mathrm{b}}(s, p, q, X)$, of Definition 2.1. We continue to call them Lipschitz-type spaces.

Definition 2.3. Let $0<p, q \leqslant \infty, s>0$ and $C_{3}>0$ be a positive constant. A real-valued function $f$ defined $\mu$-almost everywhere on $X$ belongs to the Lipschitz-type space $L_{\mathrm{t}}(s, p, q, X)$ if $f \in L^{p}(X)$ and

$$
\|f\|_{\dot{L}_{\mathrm{t}}(s, p, q, X)}=\left\|\left\{\sum_{k=0}^{\infty} 2^{k s q}\left[2^{k d} \int_{B\left(\cdot, C_{3} 2^{-k}\right)}|f(y)-f(\cdot)| \mathrm{d} \mu(y)\right]^{q}\right\}^{1 / q}\right\|_{L^{p}(X)}<\infty .
$$

Moreover, we define

$$
\|f\|_{L_{\mathrm{t}}(s, p, q, X)}=\|f\|_{L^{p}(X)}+\|f\|_{\dot{L}_{\mathrm{t}}(s, p, q, X)} .
$$

Following Definition 2.1 (see also [30]), it is easy to see that Definition 2.3 is independent of $C_{3}>0$. Moreover, if $p=q \in(0, \infty]$ and $s>0$, then

$$
L_{\mathrm{t}}(s, p, q, X)=L_{\mathrm{b}}(s, p, q, X) .
$$


As is the case with the Lipschitz norms $L(s, p, q, X)$ and $L_{\mathrm{b}}(s, p, q, X)$, the continuous version of $\|\cdot\|_{L_{\mathrm{t}}(s, p, q, X)}$ on $\mathbb{R}^{n}$ is equivalent to quasi-norms of Triebel-Lizorkin spaces $F_{p q}^{s}\left(\mathbb{R}^{n}\right)$ provided $1 \leqslant p<\infty, 0<q \leqslant \infty$ and

$$
1>s>\max \left\{0, n\left(\frac{1}{q}-\frac{1}{p}\right)\right\}
$$

(see Theorem 3.5.1 in [45, p. 187]). Again, another main aim of this paper is to prove, in Theorem 3.12 below, that this is also true on spaces of homogeneous type.

We have the following analogue of Proposition 2.2 although it should be noted that (iv) is different.

\section{Proposition 2.4.}

(i) If $s>0$ and $0<p, q \leqslant \infty$, then $L_{\mathrm{t}}(s, p, q, X)$ is a quasi-Banach space.

(ii) If $0<q_{0} \leqslant q_{1} \leqslant \infty, 0<p \leqslant \infty$ and $s>0$, then

$$
L_{\mathrm{t}}\left(s, p, q_{0}, X\right) \subset L_{\mathrm{t}}\left(s, p, q_{1}, X\right) .
$$

(iii) If $0<q_{0}, q_{1} \leqslant \infty, 0<p \leqslant \infty, s>0$ and $\epsilon>0$, then

$$
L_{\mathrm{t}}\left(s+\epsilon, p, q_{0}, X\right) \subset L_{\mathrm{t}}\left(s, p, q_{1}, X\right) .
$$

(iv) If $s>0$ and $0<p, q \leqslant \infty$, then

$$
L_{\mathrm{b}}(s, p, \min (p, q), X) \subset L_{\mathrm{t}}(s, p, q, X) \subset L_{\mathrm{b}}(s, p, \max (p, q), X) .
$$

Proof. The proofs of (i), (ii) and (iii) are similar to those of Proposition 2.2; while (iv) is a simple corollary of Proposition 13.6 in [47, p. 75] (see also Proposition 2.3 in $[48$, p. 11]).

This finishes the proof of Proposition 2.4.

The case $p=2$ of the following embedding theorem was obtained in Theorem 4.3 (iii) in [18] and Theorem 8.1 in [16] (see also Theorem 9.2 in [16]), and our proof is a slight variant of the proofs in $[\mathbf{1 6}, \mathbf{1 8}]$. See also Theorem 3.13 of $[\mathbf{4 3}]$ for some related results.

Proposition 2.5. Let $1 \leqslant p \leqslant \infty, s>d / p$ and $\lambda=s-d / p$. Then there exists a constant $C>0$ such that for all $f \in L_{\mathrm{loc}}^{1}(X)$,

$$
\|f\|_{\dot{L}(\lambda, X)} \leqslant C\|f\|_{\dot{L}_{\mathrm{b}}(s, p, \infty, X)} .
$$

Proof. Without loss of generality, we may assume that $C_{1}=1$ in the definition of the norm $\|\cdot\|_{\dot{L}(\lambda, X)}$. For $\nu \in \mathbb{Z}_{+}$and $x \in X$, we set $B_{\nu}=B\left(x, 2^{-\nu}\right)$ and

$$
f_{\nu}(x)=\frac{1}{\mu\left(B\left(x, 2^{-\nu}\right)\right)} \int_{B\left(x, 2^{-\nu}\right)} f(\xi) \mathrm{d} \mu(\xi) .
$$


By Hölder's inequality, for any $x \in X$ and $\nu \in \mathbb{Z}_{+}$, we have

$$
\begin{aligned}
\left|f_{\nu-1}(x)-f_{\nu}(x)\right|^{p} & =\left\{\frac{1}{\mu\left(B_{\nu}\right)} \frac{1}{\mu\left(B_{\nu-1}\right)}\left|\int_{B_{\nu}} \int_{B_{\nu-1}}(f(\xi)-f(\eta)) \mathrm{d} \mu(\xi) \mathrm{d} \mu(\eta)\right|\right\}^{p} \\
& \leqslant \frac{1}{\mu\left(B_{\nu}\right)} \int_{B_{\nu}}\left[\frac{1}{\mu\left(B_{\nu-1}\right)} \int_{B_{\nu-1}}|f(\xi)-f(\eta)| \mathrm{d} \mu(\xi)\right]^{p} \mathrm{~d} \mu(\eta) \\
& \leqslant \frac{C}{\mu\left(B_{\nu}\right)} \int_{X}\left[2^{\nu d} \int_{B\left(\eta, C 2^{\nu}\right)}|f(\xi)-f(\eta)| \mathrm{d} \mu(\xi)\right]^{p} \mathrm{~d} \mu(\eta) \\
& \leqslant C 2^{\nu(d-p s)}\|f\|_{\dot{L}_{\mathrm{b}}(s, p, \infty, X)}^{p}
\end{aligned}
$$

Similarly, we can prove that for any $x, y \in X$,

$$
\left|f_{\nu-1}(x)-f_{\nu}(y)\right| \leqslant C 2^{-\nu \lambda}\|f\|_{\dot{L}_{\mathrm{b}}(s, p, \infty, X)} .
$$

Thus, $\lim _{\nu \rightarrow \infty} f_{\nu}(x)$ exists everywhere and it equals $f(x) \mu$-almost everywhere. By the definition of the norm $\|\cdot\|_{\dot{L}_{\mathrm{b}}(s, p, \infty, X)}$, we can modify the definition of $f$ on a set of measure zero, without changing its norm, so that $\lim _{\nu \rightarrow \infty} f_{\nu}(x)=f(x)$ everywhere. Therefore, from (2.1) and (2.2), it follows that for any $x, y \in X$ and $r=\rho(x, y) \sim 2^{-\nu_{0}}$ with some $\nu_{0} \in \mathbb{Z}_{+}$,

$$
\left|f_{\nu_{0}}(x)-f_{\nu_{0}}(y)\right| \leqslant C r^{\lambda}\|f\|_{\dot{L}_{\mathrm{b}}(s, p, \infty, X)} .
$$

Thus, for any $x, y \in X$ and $r=\rho(x, y) \sim 2^{-\nu_{0}}$ with some $\nu_{0} \in \mathbb{Z}_{+}$, by the fact that $\lambda>0$, we have

$$
\begin{aligned}
\left|f(x)-f_{\nu_{0}}(x)\right| & =\lim _{\nu \rightarrow \infty}\left|f_{\nu+\nu_{0}}(x)-f_{\nu_{0}}(x)\right| \\
& \leqslant \sum_{k=\nu_{0}}^{\infty}\left|f_{k}(x)-f_{k+1}(x)\right| \\
& \leqslant C \sum_{k=\nu_{0}}^{\infty} 2^{-k \lambda}\|f\|_{\dot{L}_{\mathrm{b}}(s, p, \infty, X)} \\
& =C 2^{-\nu_{0} \lambda}\|f\|_{\dot{L}_{\mathrm{b}}(s, p, \infty, X)} \\
& \leqslant C r^{\lambda}\|f\|_{\dot{L}_{\mathrm{b}}(s, p, \infty, X)} .
\end{aligned}
$$

Similarly, we can verify that

$$
\left|f(y)-f_{\nu_{0}}(y)\right| \leqslant C r^{\lambda}\|f\|_{\dot{L}_{\mathrm{b}}(s, p, \infty, X)} .
$$

Estimates (2.3)-(2.5) tell us that

$$
|f(x)-f(y)| \leqslant C \rho(x, y)^{\lambda}\|f\|_{\dot{L}_{\mathrm{b}}(s, p, \infty, X)} .
$$

This completes the proof of Proposition 2.5. 


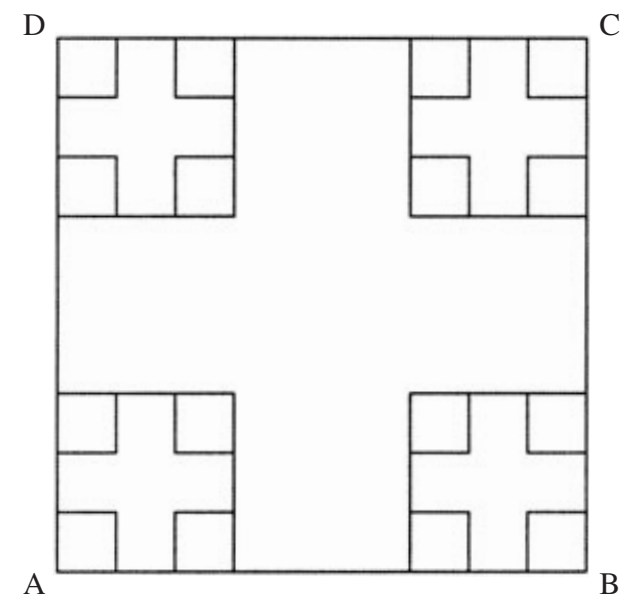

Figure 1. A self-similar set in $\mathbb{R}^{2}$.

Let $s>0$. Below we define the space $\dot{L}(s, X)$ by

$$
\dot{L}(s, X)=\left\{f \in L_{\mathrm{loc}}^{1}(X):\|f\|_{\dot{L}(s, X)}<\infty\right\} .
$$

From this proposition, Proposition 2.2 and Proposition 2.4, it is easy to deduce the following corollary.

Corollary 2.6. If $1 \leqslant p \leqslant \infty, 0<q \leqslant \infty, s>d / p$ and $\lambda=s-d / p$, then $L(s, p, q, X) \subset$ $L_{\mathrm{b}}(s, p, q, X) \subset \dot{L}(\lambda, X)$ and $L_{\mathrm{t}}(s, p, q, X) \subset \dot{L}(\lambda, X)$.

To finish this section, let us recall that if $X=\mathbb{R}^{n}$ and $s>1$, it is easy to see that the spaces $L(s, X), L_{\mathrm{b}}(s, p, q, X)$ and $L_{\mathrm{t}}(s, p, q, X)$ contain only constants, when $0<$ $p, q \leqslant \infty$. However, it is a well-known fact that this is not true if $X$ is just a space of homogeneous type, or a fractal, as is mentioned in the introduction. A simple example for the one-dimensional case was given in $[\mathbf{3 0}]$. We give another example for dimensions greater than 1 here.

Proposition 2.7. There is a self-similar set $X$ in $\mathbb{R}^{2}$ with Hausdorff dimension $d>1$ and a Lipschitz function of exponent $d$ defined on $X$.

Proof. We first construct the self-similar set $X$. Suppose that the set $E$ is the usual Cantor middle thirds set in $[0,1]$ (see $[\mathbf{3 7}]$ ). As is well known, $E$ is a self-similar set by taking away the middle part of a cell interval in every step. Let $X=E \times E$. Then $X$ is also a self-similar set with Hausdorff dimension $d=\log 4 / \log 3$. Moreover, if we let $\mu=\mathcal{H}^{d}$, the $d$-dimensional Hausdorff measure, and $\rho$ be the usual Euclidean metric, then $\left(X, \rho, \mathcal{H}^{d}\right)_{d, 1}$ is a space of homogeneous type as in Definition 1.1. This set is also an Ahlfors regular metric space. The space $X$ can also be constructed as in figure 1,

$$
X=\bigcap_{n=0}^{\infty} \bigcup_{k=1}^{4^{n}} X_{k}^{n},
$$


where $X_{1}^{0}$ is the unit square $\mathrm{ABCD}, X_{k}^{1}, k=1,2,3,4$, are four squares in the corners of $X_{1}^{0}$ with side length $\frac{1}{3}$ and so on. We obtain $X_{k}^{n}$ after $n$ steps.

Now we can define a Lipschitz function with exponent $d$ on $X$. Let $f: X \rightarrow R$ be defined by

$$
f(x)=\mathcal{H}^{d}(X \cap B(A,|x-A|)),
$$

where $|x-A|$ is the Euclidean distance between two points $x$ and $A$. For $x, y \in X$ with $x \neq y$, suppose $X_{k_{1}}^{n_{1}}$ is the minimal square containing them both; then $|x-y| \geqslant C\left(\frac{1}{3}\right)^{n_{1}}$, where the constant $C$ is independent of $x, y$ and $n_{1}$. From the Ahlfors regular property of the set $X,(1.1)$ holds with $\mu=\mathcal{H}^{d}$. Using this, together with the decreasing property of the function $x^{d}$ on $x$, we can conclude that

$$
\begin{aligned}
|f(x)-f(y)| & =\left|\mathcal{H}^{d}(X \cap B(A,|x-A|))-\mathcal{H}^{d}(X \cap B(A,|y-A|))\right| \\
& \leqslant C \mathcal{H}^{d}\left(X \cap X_{k_{1}}^{n_{1}}\right) \\
& \leqslant C\left(\frac{1}{3}\right)^{d n_{1}} \\
& \leqslant C|x-y|^{d} .
\end{aligned}
$$

Thus, $f \in L(d, X)$ and $d>1$. This completes the proof of Proposition 2.7.

Remark 2.8. From the definitions of $L(s, p, q, X), L_{\mathrm{b}}(s, p, q, X)$ and $L_{\mathrm{t}}(s, p, q, X)$, it is easy to deduce that when $\mu(X)<\infty$,

$$
L(d, X) \subset L(s, p, q, X) \cap L_{\mathrm{b}}(s, p, q, X) \cap L_{\mathrm{t}}(s, p, q, X)
$$

if $s<d$ and $0<p, q \leqslant \infty$. Thus, from Proposition 2.7, it follows that the spaces $L(s, p, q, X), L_{\mathrm{b}}(s, p, q, X)$ and $L_{\mathrm{t}}(s, p, q, X)$ may not be trivial even when $s>1$.

\section{Relations with Besov and Triebel-Lizorkin spaces}

We first recall the definition of Besov and Triebel-Lizorkin spaces on spaces of homogeneous type. To this end, we now recall the definition of the spaces of test functions on $X$ in $[\mathbf{2 2}]$ (see also [21]).

Definition 3.1. Fix $\gamma>0$ and $\theta \geqslant \beta>0$. A function $f$ defined on $X$ is said to be a test function of type $\left(x_{0}, r, \beta, \gamma\right)$ with $x_{0} \in X$ and $r>0$, if $f$ satisfies the following conditions:

(i) $|f(x)| \leqslant C \frac{r^{\gamma}}{\left(r+\rho\left(x, x_{0}\right)\right)^{d+\gamma}}$;

(ii) $|f(x)-f(y)| \leqslant C\left(\frac{\rho(x, y)}{r+\rho\left(x, x_{0}\right)}\right)^{\beta} \frac{r^{\gamma}}{\left(r+\rho\left(x, x_{0}\right)\right)^{d+\gamma}}$ for $\rho(x, y) \leqslant \frac{1}{2 A}\left[r+\rho\left(x, x_{0}\right)\right]$.

If $f$ is a test function of type $\left(x_{0}, r, \beta, \gamma\right)$, we write $f \in \mathcal{G}\left(x_{0}, r, \beta, \gamma\right)$, and the norm of $f$ in $\mathcal{G}\left(x_{0}, r, \beta, \gamma\right)$ is defined by

$$
\|f\|_{\mathcal{G}\left(x_{0}, r, \beta, \gamma\right)}=\inf \{C:(\mathrm{i}) \text { and (ii) hold }\} .
$$

Here and in what follows, $\theta$ is as in (1.2). 
Now fix $x_{0} \in X$ and let $\mathcal{G}(\beta, \gamma)=\mathcal{G}\left(x_{0}, 1, \beta, \gamma\right)$. It is easy to see that

$$
\mathcal{G}\left(x_{1}, r, \beta, \gamma\right)=\mathcal{G}(\beta, \gamma)
$$

with equivalent norms for all $x_{1} \in X$ and $r>0$. Furthermore, the space $\mathcal{G}(\beta, \gamma)$ is a Banach space with respect to the norm in $\mathcal{G}(\beta, \gamma)$. Let the dual space $(\mathcal{G}(\beta, \gamma))^{\prime}$ be the set of all linear functionals $\mathcal{L}$ from $\mathcal{G}(\beta, \gamma)$ to $\mathbb{C}$ with the property that there exists a finite constant $C \geqslant 0$ such that for all $f \in \mathcal{G}(\beta, \gamma)$,

$$
|\mathcal{L}(f)| \leqslant C\|f\|_{\mathcal{G}(\beta, \gamma)} .
$$

We denote by $\langle h, f\rangle$ the natural pairing of elements $h \in(\mathcal{G}(\beta, \gamma))^{\prime}$ and $f \in \mathcal{G}(\beta, \gamma)$. It is easy to see that $f \in \mathcal{G}\left(x_{0}, r, \beta, \gamma\right)$ with $x_{0} \in X$ and $r>0$ if and only if $f \in \mathcal{G}(\beta, \gamma)$. Thus, for all $h \in(\mathcal{G}(\beta, \gamma))^{\prime},\langle h, f\rangle$ is well defined for all $f \in \mathcal{G}\left(x_{0}, r, \beta, \gamma\right)$ with $x_{0} \in X$ and $r>0$. Below we denote by $\mathcal{G}(\beta, \gamma)$, for $0<\beta, \gamma<\theta$, the completion of $\mathcal{G}(\theta, \theta)$ in $\mathcal{G}(\beta, \gamma)$.

To state the definition of the inhomogeneous Besov spaces $B_{p q}^{s}(X)$ and the inhomogeneous Triebel-Lizorkin spaces $F_{p q}^{s}(X)$ studied in [23-26], we need the following approximations to the identity, first introduced in $[\mathbf{2 1}]$.

Definition 3.2. A sequence $\left\{S_{k}\right\}_{k=0}^{\infty}$ of linear operators is said to be an approximation to the identity of order $\epsilon \in(0, \theta]$ if there exist constants $C_{4}, C_{5}>0$ such that for all $k \in \mathbb{Z}_{+}$and all $x, x^{\prime}, y$ and $y^{\prime} \in X, S_{k}(x, y)$, the kernel of $S_{k}$, is a function from $X \times X$ into $\mathbb{C}$ satisfying

(i) $S_{k}(x, y)=0$ if $\rho(x, y) \geqslant C_{4} 2^{-k}$ and $\left\|S_{k}\right\|_{L^{\infty}(X \times X)} \leqslant C_{5} 2^{d k}$;

(ii) $\left|S_{k}(x, y)-S_{k}\left(x^{\prime}, y\right)\right| \leqslant C_{5} 2^{k(d+\epsilon)} \rho\left(x, x^{\prime}\right)^{\epsilon}$;

(iii) $\left|S_{k}(x, y)-S_{k}\left(x, y^{\prime}\right)\right| \leqslant C_{5} 2^{k(d+\epsilon)} \rho\left(y, y^{\prime}\right)^{\epsilon}$;

(iv) $\left|\left[S_{k}(x, y)-S_{k}\left(x, y^{\prime}\right)\right]-\left[S_{k}\left(x^{\prime}, y\right)-S_{k}\left(x^{\prime}, y^{\prime}\right)\right]\right| \leqslant C_{5} 2^{k(d+2 \epsilon)} \rho\left(x, x^{\prime}\right)^{\epsilon} \rho\left(y, y^{\prime}\right)^{\epsilon}$;

(v) $\int_{X} S_{k}(x, y) \mathrm{d} \mu(y)=1$;

(vi) $\int_{X} S_{k}(x, y) \mathrm{d} \mu(x)=1$.

Here, to say that $S_{k}(x, y)$ is the kernel of $S_{k}$ means that for suitable functions $f$,

$$
S_{k} f(x)=\int_{X} S_{k}(x, y) f(y) \mathrm{d} \mu(y) .
$$

We point out that by a construction similar to Coifman's one in [8], one can construct an approximation to the identity with compact support as in Definition 3.2, for spaces of homogeneous type.

We also need the following construction due to Christ [6], which provides an analogue of the grid of Euclidean dyadic cubes on a space of homogeneous type.

Lemma 3.3. Let $(X, \rho, \mu)_{d, \theta}$ be a space of homogeneous type. Then there exists a collection $\left\{Q_{\alpha}^{k} \subset X: k \in \mathbb{Z}_{+}, \alpha \in I_{k}\right\}$ of open subsets, where $I_{k}$ is some (possibly finite) 
index set, and constants $\delta \in(0,1), C_{6}>0$ and $C_{7}>0$ such that

(i) $\mu\left(X \backslash \cup_{\alpha} Q_{\alpha}^{k}\right)=0$ for each fixed $k$ and $Q_{\beta}^{k} \cap Q_{\alpha}^{k}=\emptyset$ if $\alpha \neq \beta$;

(ii) for any $\alpha, \beta, k, l$ with $l \geqslant k$, either $Q_{\beta}^{l} \subset Q_{\alpha}^{k}$ or $Q_{\beta}^{l} \cap Q_{\alpha}^{k}=\emptyset$;

(iii) for each $(k, \alpha)$ and each $l<k$ there is a unique $\beta$ such that $Q_{\alpha}^{k} \subset Q_{\beta}^{l}$;

(iv) $\operatorname{diam}\left(Q_{\alpha}^{k}\right) \leqslant C_{6} \delta^{k}$;

(v) each $Q_{\alpha}^{k}$ contains some ball $B\left(z_{\alpha}^{k}, C_{7} \delta^{k}\right)$, where $z_{\alpha}^{k} \in X$.

In fact, we can think of $Q_{\alpha}^{k}$ as being essentially a cube of diameter approximately $\delta^{k}$ with centre $z_{\alpha}^{k}$. We will always suppose that $\delta=\frac{1}{2}$, although, in fact, this restriction can be removed (see [22]). We denote by $Q_{\tau}^{k, \nu}, \nu=1,2, \ldots, N(k, \tau)$, the set of all cubes $Q_{\tau^{\prime}}^{k+j} \subset Q_{\tau}^{k}$, where $j$ is a fixed large positive integer. Denote by $y_{\tau}^{k, \nu}$ a point in $Q_{\tau}^{k, \nu}$. For any dyadic cube $Q$ and any $f \in L_{\text {loc }}^{1}(X)$, we set

$$
m_{Q}(f)=\frac{1}{\mu(Q)} \int_{Q} f(x) \mathrm{d} \mu(x) .
$$

We also let $a_{+}=\max (a, 0)$ for any $a \in \mathbb{R}$.

Now we can state the definition of Besov spaces $B_{p q}^{s}(X)$ and Triebel-Lizorkin spaces $F_{p q}^{s}(X)$ from $[\mathbf{2 4}]$.

Definition 3.4. Let $-\theta<s<\theta,\left\{S_{k}\right\}_{k=0}^{\infty}$ be as in Definition 3.2 with order $\theta, D_{0}=S_{0}$ and $D_{k}=S_{k}-S_{k-1}$ for $k \in \mathbb{N}$. Suppose that $\beta$ and $\gamma$ satisfy

$$
\max \left(0,-s+d(1 / p-1)_{+}\right)<\beta<\theta \text { and } 0<\gamma<\theta .
$$

Let $j \in \mathbb{N}$ be fixed and sufficiently large, and $\left\{Q_{\tau}^{0, \nu}: \tau \in I_{0}, \nu=1, \ldots, N(0, \tau)\right\}$ be as above.

(i) The inhomogeneous Besov space $B_{p q}^{s}(X)$, defined for

$$
\max \left(\frac{d}{d+\theta}, \frac{d}{d+\theta+s}\right)<p \leqslant \infty
$$

and $0<q \leqslant \infty$, is the collection of all $f \in(\stackrel{\mathcal{G}}{(\beta, \gamma}, \gamma))^{\prime}$ with given $\beta$ and $\gamma$ satisfying (3.1), such that

$$
\begin{aligned}
\|f\|_{B_{p q}^{s}(X)}=\left\{\sum_{\tau \in I_{0}} \sum_{\nu=1}^{N(0, \tau)} \mu\left(Q_{\tau}^{0, \nu}\right)\left[m_{Q_{\tau}^{0, \nu}}\left(\left|D_{0}(f)\right|\right)\right]^{p}\right\}^{1 / p} & \\
& +\left\{\sum_{k=1}^{\infty}\left[2^{k s}\left\|D_{k}(f)\right\|_{L^{p}(X)}\right]^{q}\right\}^{1 / q}<\infty .
\end{aligned}
$$


(ii) The inhomogeneous Triebel-Lizorkin space $F_{p q}^{s}(X)$, defined for

$$
\max \left(\frac{d}{d+\theta}, \frac{d}{d+\theta+s}\right)<p<\infty
$$

and $\max (d /(d+\theta), d /(d+\theta+s))<q \leqslant \infty$, is the collection of all $f \in(\mathcal{G}(\beta, \gamma))^{\prime}$ with given $\beta$ and $\gamma$ satisfying (3.1), such that

$$
\begin{aligned}
\|f\|_{F_{p q}^{s}(X)}=\left\{\sum_{\tau \in I_{0}} \sum_{\nu=1}^{N(0, \tau)} \mu\left(Q_{\tau}^{0, \nu}\right)\left[m_{Q_{\tau}^{0, \nu}}\left(\left|D_{0}(f)\right|\right)\right]^{p}\right\}^{1 / p} & \\
& +\left\|\left\{\sum_{k=1}^{\infty}\left[2^{k s}\left|D_{k}(f)\right|\right]^{q}\right\}^{1 / q}\right\|_{L^{p}(X)}<\infty .
\end{aligned}
$$

It was proved in $[\mathbf{2 4}]$ that Definition 3.4 is independent of the choices of large positive integers $j$, approximations to the identity and the pairs $(\beta, \gamma)$ in $(3.1)$.

One of the main aims of this section is to establish the connection between the Lipschitz-type spaces defined in $\S 2$ and the Besov spaces defined in [25] and [24].

Proposition 3.5. If $0<s<\theta, 1 \leqslant p \leqslant \infty$ and $0<q \leqslant \infty$, then

$$
L(s, p, q, X) \subset L_{\mathrm{b}}(s, p, q, X) \subset B_{p q}^{s}(X) .
$$

Moreover, there is a constant $C>0$ such that

$$
\|f\|_{B_{p q}^{s}(X)} \leqslant C\|f\|_{L_{\mathrm{b}}(s, p, q, X)} \leqslant C\|f\|_{L(s, p, q, X)}
$$

for all $f \in L(s, p, q, X)$ and all $f \in L_{\mathrm{b}}(s, p, q, X)$.

Proof. By Proposition 2.2 (ii), we need only show that $L_{\mathrm{b}}(s, p, q, X) \subset B_{p q}^{s}(X)$, and

$$
\|f\|_{B_{p q}^{s}(X)} \leqslant C\|f\|_{L_{\mathrm{b}}(s, p, q, X)}
$$

for all $f \in L_{\mathrm{b}}(s, p, q, X)$. Let $f \in L_{\mathrm{b}}(s, p, q, X)$ and $\left\{D_{k}\right\}_{k \in \mathbb{Z}_{+}}$be as in Definition 3.4. Since $\mu\left(Q_{\tau}^{0, \nu}\right) \sim C$, the Hölder inequality and the support property of $D_{0}$ imply that

$$
\begin{aligned}
\left\{\sum_{\tau \in I_{0}} \sum_{\nu=1}^{N(0, \tau)} \mu\left(Q_{\tau}^{0, \nu}\right)\left[m_{Q_{\tau}^{0, \nu}}\left(\left|D_{0}(f)\right|\right)\right]^{p}\right\}^{1 / p} & \leqslant C\left\{\sum_{\tau \in I_{0}} \sum_{\nu=1}^{N(0, \tau)} \int_{Q_{\tau}^{0, \nu}}\left|D_{0}(f)(x)\right|^{p} \mathrm{~d} \mu(x)\right\}^{1 / p} \\
& \leqslant C\left\{\int_{X}\left|D_{0}(f)(x)\right|^{p} \mathrm{~d} \mu(x)\right\}^{1 / p} \\
& \leqslant C\left\{\int_{X} \int_{X}\left|D_{0}(x, y)\right||f(y)|^{p} \mathrm{~d} \mu(y) \mathrm{d} \mu(x)\right\}^{1 / p} \\
& \leqslant C\|f\|_{L^{p}(X)} \\
& \leqslant C\|f\|_{L_{\mathrm{b}}(s, p, q, X) .}
\end{aligned}
$$


By the size condition, the vanishing moment property and the support condition of $D_{k}$ when $k \in \mathbb{N}$, it follows that

$$
\begin{aligned}
\left\{\sum_{k=1}^{\infty}\left[2^{k s}\left\|D_{k}(f)\right\|_{L^{p}(X)}\right]^{q}\right\}^{1 / q} & \\
= & \left\{\sum_{k=1}^{\infty} 2^{k s q}\left[\int_{X}\left(\int_{X} D_{k}(x, y)[f(y)-f(x)] \mathrm{d} \mu(y)\right)^{p} \mathrm{~d} \mu(x)\right]^{q / p}\right\}^{1 / q} \\
& \leqslant\left\{\sum_{k=1}^{\infty} 2^{k s q}\left(\int_{X}\left[2^{k d} \int_{B\left(x, C 2^{-k}\right)}|f(y)-f(x)| \mathrm{d} \mu(y)\right]^{p} \mathrm{~d} \mu(x)\right)^{q / p}\right\}^{1 / q} \\
& \leqslant C\|f\|_{L_{\mathrm{b}}(s, p, q, X)} .
\end{aligned}
$$

Combining (3.2), (3.3) with Definitions 3.4 and 2.1 yields that

$$
\|f\|_{B_{p q}^{s}(X)} \leqslant C\|f\|_{L_{\mathrm{b}}(s, p, q, X)},
$$

where $C$ is independent of $f$. This implies that $L_{\mathrm{b}}(s, p, q, X) \subset B_{p q}^{s}(X)$ and completes the proof of Proposition 3.5.

To establish the converse of Proposition 3.5, we need the following discrete Calderón reproducing formula from $[\mathbf{2 7}]$.

Lemma 3.6. Suppose that $\left\{D_{k}\right\}_{k=0}^{\infty}$ is as in Definition 3.4. Then there exist functions $\tilde{D}_{Q_{\tau}^{0, \nu}}$ with $\tau \in I_{0}$ and $\nu=1, \ldots, N(0, \tau)$ and $\tilde{D}_{k}(x, y)$ with $k \in \mathbb{N}$ such that for any fixed $y_{\tau}^{k, \nu} \in Q_{\tau}^{k, \nu}$ with $k \in \mathbb{N}, \tau \in I_{k}$ and $\nu \in\{1, \ldots, N(k, \tau)\}$ and all $f \in\left(\stackrel{\mathcal{G}}{(}\left(\beta_{1}, \gamma_{1}\right)\right)^{\prime}$ with $0<\beta_{1}<\theta$ and $0<\gamma_{1}<\theta$,

$$
\begin{aligned}
f(x)=\sum_{\tau \in I_{0}} \sum_{\nu=1}^{N(0, \tau)} \mu\left(Q_{\tau}^{0, \nu}\right) m_{Q_{\tau}^{0, \nu}}\left(D_{0}(f)\right) \tilde{D}_{Q_{\tau}^{0, \nu}}(x) & \\
& +\sum_{k=1}^{\infty} \sum_{\tau \in I_{k}} \sum_{\nu=1}^{N(k, \tau)} \mu\left(Q_{\tau}^{k, \nu}\right) D_{k}(f)\left(y_{\tau}^{k, \nu}\right) \tilde{D}_{k}\left(x, y_{\tau}^{k, \nu}\right) .
\end{aligned}
$$

Here the series converge in $\left.\left(\stackrel{\mathcal{G}}{(} \beta_{1}^{\prime}, \gamma_{1}^{\prime}\right)\right)^{\prime}$ for $\beta_{1}<\beta_{1}^{\prime}<\theta$ and $\gamma_{1}<\gamma_{1}^{\prime}<\theta ; \tilde{D}_{k}(x, y)$ with $k \in \mathbb{N}$ satisfies that for any given $\epsilon \in(0, \theta)$,

(i) $\left|\tilde{D}_{k}(x, y)\right| \leqslant C \frac{2^{-k \epsilon}}{\left(2^{-k}+\rho(x, y)\right)^{d+\epsilon}}$,

(ii) $\left|\tilde{D}_{k}(x, y)-\tilde{D}_{k}\left(x^{\prime}, y\right)\right| \leqslant C\left(\frac{\rho\left(x, x^{\prime}\right)}{2^{-k}+\rho(x, y)}\right)^{\epsilon} \frac{2^{-k \epsilon}}{\left(2^{-k}+\rho(x, y)\right)^{d+\epsilon}}$

$$
\text { for } \rho\left(x, x^{\prime}\right) \leqslant \frac{1}{2 A}(1+\rho(x, y)) \text {, }
$$

(iii) $\int_{X} \tilde{D}_{k}(x, y) \mathrm{d} \mu(x)=\int_{X} \tilde{D}_{k}(x, y) \mathrm{d} \mu(y)=0$; 
$\operatorname{diam}\left(Q_{\tau}^{0, \nu}\right) \sim 2^{-j}$ for $\tau \in I_{0}$ and $\nu=1, \ldots, N(0, \tau)$ with some $j \in \mathbb{N} ; \tilde{D}_{Q_{\tau}^{0, \nu}}(x)$ for $\tau \in I_{0}$ and $\nu=1, \ldots, N(0, \tau)$ satisfies that

(iv) $\int_{X} \tilde{D}_{Q_{\tau}^{0, \nu}}(x) \mathrm{d} \mu(x)=1$,

(v) for any given $\epsilon \in(0, \theta)$, there is a constant $C>0$ such that

$$
\left|\tilde{D}_{Q_{\tau}^{0, \nu}}(x)\right| \leqslant C \frac{1}{(1+\rho(x, y))^{d+\epsilon}}
$$

for all $x \in X$ and $y \in Q_{\tau}^{0, \nu}$, and

(vi)

$$
\left|\tilde{D}_{Q_{\tau}^{0, \nu}}(x)-\tilde{D}_{Q_{\tau}^{0, \nu}}(z)\right| \leqslant C\left(\frac{\rho(x, z)}{1+\rho(x, y)}\right)^{\epsilon} \frac{1}{(1+\rho(x, y))^{d+\epsilon}}
$$

for all $x, z \in X$ and all $y \in Q_{\tau}^{0, \nu}$ satisfying $\rho(x, z) \leqslant(1 / 2 A)(1+\rho(x, y))$.

Moreover, $j$ can be any fixed large positive integer and the constant $C$ in (v) and (vi) is independent of $j$.

Proposition 3.7. Let $0<s<\theta$ and $0<q \leqslant \infty$. Then

(i) if $d /(d+\theta)<p \leqslant \infty$, then $B_{p q}^{s}(X) \subset L(s, p, q, X)$ and, moreover, there is a constant $C>0$ such that for all $f \in B_{p q}^{s}(X)$,

$$
\|f\|_{L(s, p, q, X)} \leqslant C\|f\|_{B_{p q}^{s}(X)}
$$

(ii) if $1 \leqslant p \leqslant \infty$, then $B_{p q}^{s}(X) \subset L_{\mathrm{b}}(s, p, q, X)$ and, moreover, there is a constant $C>0$ such that for all $f \in B_{p q}^{s}(X)$,

$$
\|f\|_{L_{\mathrm{b}}(s, p, q, X)} \leqslant C\|f\|_{B_{p q}^{s}(X)} .
$$

Proof. (ii) is a simple corollary of (i) and Proposition 2.2 (ii). Thus, we need only prove (i). We first point out that by the definition of the space $B_{p q}^{s}(X)$ and the Hölder inequality, it is easy to see that

$$
B_{p q}^{s}(X) \subset B_{p, \min (p, q)}^{s / 2}(X) \subset F_{p q}^{s / 2}(X) \subset F_{p 2}^{0}(X)
$$

(see also Proposition 1.2 and (5.1) in [23], Proposition 2 in [44, p. 47] and Proposition 13.6 in $[\mathbf{4 8}])$. If $1<p<\infty$, then by Theorem 3 in [27], we obtain

$$
F_{p 2}^{0}(X)=L^{p}(X)
$$


From (3.5) and (3.6) it follows that if $p \in(1, \infty)$, then

$$
\|f\|_{L^{p}(X)} \leqslant C\|f\|_{B_{p q}^{s}(X)} .
$$

We now show that (3.7) also holds when $p=\infty$. To do so, decompose $f$ as in (3.4) and fix $\epsilon \in(0, \theta)$. By the size condition (v) on $\tilde{D}_{Q_{\tau}^{0, \nu}}$, the size condition (i) on $\tilde{D}_{k}$ given by Lemma 3.6 and the fact that $y_{\tau}^{k, \nu}$ is arbitrary, we have

$$
\begin{aligned}
& |f(x)| \leqslant \sum_{\tau \in I_{0}} \sum_{\nu=1}^{N(0, \tau)} \mu\left(Q_{\tau}^{0, \nu}\right) m_{Q_{\tau}^{0, \nu}}\left(\left|D_{0}(f)\right|\right)\left|\tilde{D}_{Q_{\tau}^{0, \nu}}(x)\right| \\
& +\sum_{k=1}^{\infty} \sum_{\tau \in I_{0}} \sum_{\nu=1}^{N(k, \tau)} \mu\left(Q_{\tau}^{k, \nu}\right)\left|D_{k}(f)\left(y_{\tau}^{k, \nu}\right)\right|\left|\tilde{D}_{k}\left(x, y_{\tau}^{k, \nu}\right)\right| \\
& \leqslant C \sup _{\tau \in I_{0}, \nu=1, \ldots, N(0, \tau)} m_{Q_{\tau}^{0, \nu}}\left(\left|D_{0}(f)\right|\right) \sum_{\tau \in I_{0}} \sum_{\nu=1}^{N(0, \tau)} \int_{Q_{\tau}^{0, \nu}} \frac{1}{(1+\rho(x, y))^{d+\epsilon}} \mathrm{d} \mu(y) \\
& +C \sum_{k=1}^{\infty} \sup _{x \in X}\left|D_{k}(f)(x)\right| \sum_{\tau \in I_{k}} \sum_{\nu=1}^{N(k, \tau)} \int_{Q_{\tau}^{k, \nu}} \frac{2^{-k \epsilon}}{\left(2^{-k}+\rho(x, y)\right)^{d+\epsilon}} \mathrm{d} \mu(y) \\
& \leqslant C \sup _{\tau \in I_{0}, \nu=1, \ldots, N(0, \tau)} m_{Q_{\tau}^{0, \nu}}\left(\left|D_{0}(f)\right|\right) \int_{X} \frac{1}{(1+\rho(x, y))^{d+\epsilon}} \mathrm{d} \mu(y) \\
& +C \sum_{k=1}^{\infty} \sup _{x \in X}\left|D_{k}(f)(x)\right| \int_{X} \frac{2^{-k \epsilon}}{\left(2^{-k}+\rho(x, y)\right)^{d+\epsilon}} \mathrm{d} \mu(y) \\
& \leqslant C \sup _{\tau \in I_{0}, \nu=1, \ldots, N(0, \tau)} m_{Q_{\tau}^{0, \nu}}\left(\left|D_{0}(f)\right|\right)+C \sum_{k=1}^{\infty} \sup _{x \in X}\left|D_{k}(f)(x)\right| \\
& \leqslant \begin{cases}C\|f\|_{B_{\infty q}^{s}(X)}+C\left\{\sum_{k=1}^{\infty}\left[2^{k s} \sup _{x \in X}\left|D_{k}(f)(x)\right|\right]^{q}\right\}^{1 / q}, & 0<q \leqslant 1, \\
C\|f\|_{B_{\infty q}^{s}(X)}+C\left\{\sum_{k=1}^{\infty}\left[2^{k s} \sup _{x \in X}\left|D_{k}(f)(x)\right|\right]^{q}\right\}^{1 / q}\left\{\sum_{k=1}^{\infty} 2^{-k s q^{\prime}}\right\}^{1 / q^{\prime}}, \\
1<q \leqslant \infty,\end{cases} \\
& \leqslant C\|f\|_{B_{\infty q}^{s}(X)} \text {. }
\end{aligned}
$$

We have used the fact that $s>0$ and the Minkowski inequality: for $\nu \in(0,1]$,

$$
\left(\sum_{j}\left|a_{j}\right|\right)^{\nu} \leqslant \sum_{j}\left|a_{j}\right|^{\nu}
$$

with $a_{j} \in \mathbb{C}$ for all $j$. Thus, if $0<s<\theta$ and $0<q \leqslant \infty$, then

$$
\|f\|_{L^{\infty}(X)} \leqslant C\|f\|_{B_{\infty q}^{s}(X)}
$$


Let us now consider the case $p \leqslant 1$. We follow similar steps to the argument for (3.8). When $0<q / p \leqslant 1$, we write $f$ as in (3.4). By (3.9), the fact that $y_{\tau}^{k, \nu}$ is arbitrary, $\mu\left(Q_{\tau}^{0, \nu}\right) \sim C$, the size condition $(\mathrm{v})$ on $\tilde{D}_{Q_{\tau}^{0, \nu}}$ and the size condition (i) on $\tilde{D}_{k}$ from Lemma 3.6 and taking $\epsilon \in(0, \theta)$ such that $d<(d+\epsilon) p$, we have

$$
\begin{aligned}
\|f\|_{L^{p}(X)}^{p} & \\
\leqslant & C \sum_{\tau \in I_{0}} \sum_{\nu=1}^{N(0, \tau)} \mu\left(Q_{\tau}^{0, \nu}\right)^{p}\left[m_{Q_{\tau}^{0, \nu}}\left(\left|D_{0}(f)\right|\right)\right]^{p} \int_{X} \frac{1}{\left(1+\rho\left(x, y_{\tau}^{0, \nu}\right)\right)^{(d+\epsilon) p}} \mathrm{~d} \mu(x) \\
& +C \sum_{k=1}^{\infty} \sum_{\tau \in I_{k}} \sum_{\nu=1}^{N(k, \tau)} \mu\left(Q_{\tau}^{k, \nu}\right)^{p}\left|D_{k}(f)\left(y_{\tau}^{k, \nu}\right)\right|^{p} \int_{X} \frac{2^{-k \epsilon p}}{\left(2^{-k}+\rho\left(x, y_{\tau}^{k, \nu}\right)\right)^{(d+\epsilon) p}} \mathrm{~d} \mu(x) \\
\leqslant & C \sum_{\tau \in I_{0}} \sum_{\nu=1}^{N(0, \tau)} \mu\left(Q_{\tau}^{0, \nu}\right)\left[m_{Q_{\tau}^{0, \nu}}\left(\left|D_{0}(f)\right|\right)\right]^{p}+C \sum_{k=1}^{\infty} \sum_{\tau \in I_{k}} \sum_{\nu=1}^{N(k, \tau)} \mu\left(Q_{\tau}^{k, \nu}\right)\left|D_{k}(f)\left(y_{\tau}^{k, \nu}\right)\right|^{p} \\
\leqslant & C\|f\|_{B_{p q}^{s}(X)}^{p}+C \sum_{k=1}^{\infty} \sum_{\tau \in I_{k}} \sum_{\nu=1}^{N(k, \tau)} \int_{Q_{\tau}^{k, \nu}}\left|D_{k}(f)(y)\right|^{p} \mathrm{~d} \mu(y) \\
= & C\|f\|_{B_{p q}^{s}(X)}^{p}+C \sum_{k=1}^{\infty}\left\|D_{k}(f)\right\|_{L^{p}(X)}^{p} \\
\leqslant & C\|f\|_{B_{p q}^{s}(X)}^{p}+C\left\{\sum_{k=1}^{\infty}\left[2^{k s}\left\|D_{k}(f)\right\|_{L^{p}(X)}\right]^{q} 2^{-k s q}\right\}^{p / q} \\
\leqslant & C\|f\|_{B_{p q}^{s}(X)}^{p}+C\left\{\sum_{k=1}^{\infty}\left[2^{k s}\left\|D_{k}(f)\right\|_{L^{p}(X)}\right]^{q}\right\}^{p / q} \\
\leqslant & C\|f\|_{B_{p q}^{s}(X)}^{p} .
\end{aligned}
$$

When $1<q / p \leqslant \infty$, we use the Hölder inequality to obtain

$$
\begin{aligned}
\|f\|_{L^{p}(X)}^{p} & \leqslant C\|f\|_{B_{p q}^{s}(X)}^{p}+C \sum_{k=1}^{\infty}\left\|D_{k}(f)\right\|_{L^{p}(X)}^{p} \\
& \leqslant C\|f\|_{B_{p q}^{s}(X)}^{p}+C\left\{\sum_{k=1}^{\infty}\left[2^{k s}\left\|D_{k}(f)\right\|_{L^{p}(X)}\right]^{q}\right\}^{p / q}\left\{\sum_{k=1}^{\infty} 2^{-k s p(q / p)^{\prime}}\right\}^{1-p / q} \\
& \leqslant C\|f\|_{B_{p q}^{s}(X)}^{p} .
\end{aligned}
$$

Thus, if $d /(d+\theta)<p \leqslant 1$, we have

$$
\|f\|_{L^{p}(X)} \leqslant C\|f\|_{B_{p q}^{s}(X)}
$$


We now estimate the second term appearing in the definition of the norm of $f$ in $L(s, p, q, X)$. Without loss of generality, we may assume $C_{2}=1$. By (3.4), we may write

$$
\begin{aligned}
& \left\{\sum_{l=0}^{\infty} 2^{l s q}\left(2^{l d} \int_{X} \int_{B\left(x, 2^{-l}\right)}|f(x)-f(y)|^{p} \mathrm{~d} \mu(y) \mathrm{d} \mu(x)\right)^{q / p}\right\}^{1 / q} \\
& \leqslant C\left\{\sum _ { l = 0 } ^ { \infty } 2 ^ { l s q } \left(2^{l d} \int_{X} \int_{B\left(x, 2^{-l}\right)} \mid \sum_{\tau \in I_{0}} \sum_{\nu=1}^{N(0, \tau)} \mu\left(Q_{\tau}^{0, \nu}\right) m_{Q_{\tau}^{0, \nu}}\left(D_{0}(f)\right)\right.\right. \\
& \left.\left.\times\left.\left[\tilde{D}_{Q_{\tau}^{0, \nu}}(x)-\tilde{D}_{Q_{\tau}^{0, \nu}}(y)\right]\right|^{p} \mathrm{~d} \mu(y) \mathrm{d} \mu(x)\right)^{q / p}\right\}^{1 / q} \\
& +C\left\{\sum _ { l = 0 } ^ { \infty } 2 ^ { l s q } \left(2^{l d} \int_{X} \int_{B\left(x, 2^{-l}\right)} \mid \sum_{k=1}^{l+1} \sum_{\tau \in I_{k}} \sum_{\nu=1}^{N(k, \tau)} \mu\left(Q_{\tau}^{k, \nu}\right) D_{k}(f)\left(y_{\tau}^{k, \nu}\right)\right.\right. \\
& \left.\left.\quad \times\left.\left[\tilde{D}_{k}\left(x, y_{\tau}^{k, \nu}\right)-\tilde{D}_{k}\left(y, y_{\tau}^{k, \nu}\right)\right]\right|^{p} \mathrm{~d} \mu(y) \mathrm{d} \mu(x)\right)^{q / p}\right\}^{1 / q} \\
& +C\left\{\sum_{l=0}^{\infty} 2^{l s q}\left(2^{l d} \int_{X} \int_{B\left(x, 2^{-l}\right)}\left|\sum_{k=l+2}^{\infty} \sum_{\tau \in I_{k}} \sum_{\nu=1}^{N(k, \tau)} \ldots\right|^{p} \mathrm{~d} \mu(y) \mathrm{d} \mu(x)\right)^{q / p}\right\}^{1 / q} \\
& =E_{1}+E_{2}+E_{3} .
\end{aligned}
$$

For $E_{1}$ and $p \leqslant 1$, we use $(3.9)$, the fact that $\mu\left(Q_{\tau}^{0, \nu}\right) \sim C$, the regularity of $\tilde{D}_{Q_{\tau}^{0, \nu}}$ from Lemma 3.6 (iv). Choosing $\epsilon \in(s, \theta)$ such that $d<(d+\epsilon) p$, we use Lemma 3.3 and the fact that

$$
1+\rho\left(x, y_{\tau}^{0, \nu}\right) \sim 1+\rho(x, y)
$$

for any $y \in Q_{\tau}^{0, \nu}$, to estimate $E_{1}$ as follows:

$$
\begin{aligned}
& E_{1} \leqslant C\left\{\sum _ { l = 0 } ^ { \infty } 2 ^ { l s q } \left(2 ^ { l d } \int _ { X } \int _ { B ( x , 2 ^ { - l } ) } \left[\sum_{\tau \in I_{0}} \sum_{\nu=1}^{N(0, \tau)} \mu\left(Q_{\tau}^{0, \nu}\right) m_{Q_{\tau}^{0, \nu}}\left(\left|D_{0}(f)\right|\right)\right.\right.\right. \\
& \left.\left.\left.\times\left(\frac{\rho(x, y)}{1+\rho\left(x, y_{\tau}^{0, \nu}\right)}\right)^{\epsilon} \frac{1}{\left(1+\rho\left(x, y_{\tau}^{0, \nu}\right)\right)^{d+\epsilon}}\right]^{p} \mathrm{~d} \mu(y) \mathrm{d} \mu(x)\right)^{q / p}\right\}^{1 / q} \\
& \leqslant C\left\{\sum _ { l = 0 } ^ { \infty } 2 ^ { l ( s - \epsilon ) q } \left(\int _ { X } \left[\sum_{\tau \in I_{0}} \sum_{\nu=1}^{N(0, \tau)} \mu\left(Q_{\tau}^{0, \nu}\right) m_{Q_{\tau}^{0, \nu}}\left(\left|D_{0}(f)\right|\right)\right.\right.\right. \\
& \leqslant C\left\{\sum _ { l = 0 } ^ { \infty } 2 ^ { l ( s - \epsilon ) q } \left(\sum_{\tau \in I_{0}} \sum_{\nu=1}^{N(0, \tau)} \mu\left(Q_{\tau}^{0, \nu}\right)^{p}\left[m_{Q_{\tau}^{0, \nu}}\left(\left|D_{0}(f)\right|\right)\right]^{p}\right.\right. \\
& \left.\left.\left.\times \int_{X} \frac{1}{\left(1+\rho\left(x, y_{\tau}^{0, \nu}\right)\right)^{d+\epsilon}}\right]^{p} \mathrm{~d} \mu(x)\right)^{q / p}\right\}^{1 / q} \\
& \left.\left.\left.\leqslant\left(x, y_{\tau}^{0, \nu}\right)\right)^{(d+\epsilon) p} \mathrm{~d} \mu(x)\right)^{q / p}\right\}^{1 / q}
\end{aligned}
$$




$$
\begin{aligned}
& \leqslant C\left\{\sum_{l=0}^{\infty} 2^{l(s-\epsilon) q}\left(\sum_{\tau \in I_{0}} \sum_{\nu=1}^{N(0, \tau)} \mu\left(Q_{\tau}^{0, \nu}\right)\left[m_{Q_{\tau}^{0, \nu}}\left(\left|D_{0}(f)\right|\right)\right]^{p}\right)^{q / p}\right\}^{1 / q} \\
& \leqslant C\left\{\sum_{\tau \in I_{0}} \sum_{\nu=1}^{N(0, \tau)} \mu\left(Q_{\tau}^{0, \nu}\right)\left[m_{Q_{\tau}^{0, \nu}}\left(\left|D_{0}(f)\right|\right)\right]^{p}\right\}^{1 / p} \\
& \leqslant C\|f\|_{B_{p q}^{s}(X)} .
\end{aligned}
$$

On the other hand, if $1<p \leqslant \infty$, the Hölder inequality implies that

$$
\begin{aligned}
& E_{1} \leqslant C\left\{\sum _ { l = 0 } ^ { \infty } 2 ^ { l ( s - \epsilon ) q } \left(\int _ { X } \left[\sum_{\tau \in I_{0}} \sum_{\nu=1}^{N(0, \tau)} \mu\left(Q_{\tau}^{0, \nu}\right) m_{Q_{\tau}^{0, \nu}}\left(\left|D_{0}(f)\right|\right)\right.\right.\right. \\
& \left.\left.\left.\times \frac{1}{\left(1+\rho\left(x, y_{\tau}^{0, \nu}\right)\right)^{d+\epsilon}}\right]^{p} \mathrm{~d} \mu(x)\right)^{q / p}\right\}^{1 / q} \\
& \leqslant C\left\{\sum _ { l = 0 } ^ { \infty } 2 ^ { l ( s - \epsilon ) q } \left[\int_{X}\left(\sum_{\tau \in I_{0}} \sum_{\nu=1}^{N(0, \tau)} \mu\left(Q_{\tau}^{0, \nu}\right)\left[m_{Q_{\tau}^{0, \nu}}\left(\left|D_{0}(f)\right|\right)\right]^{p} \frac{1}{\left(1+\rho\left(x, y_{\tau}^{0, \nu}\right)\right)^{d+\epsilon}}\right)\right.\right. \\
& \left.\left.\times\left[\sum_{\tau \in I_{0}} \sum_{\nu=1}^{N(0, \tau)} \mu\left(Q_{\tau}^{0, \nu}\right) \frac{1}{\left(1+\rho\left(x, y_{\tau}^{0, \nu}\right)\right)^{d+\epsilon}}\right]^{p / p^{\prime}} \mathrm{d} \mu(x)\right]^{q / p}\right\}^{1 / q} \\
& \leqslant C\left\{\sum _ { l = 0 } ^ { \infty } 2 ^ { l ( s - \epsilon ) q } \left[\int_{X}\left(\sum_{\tau \in I_{0}} \sum_{\nu=1}^{N(0, \tau)} \mu\left(Q_{\tau}^{0, \nu}\right)\left[m_{Q_{\tau}^{0, \nu}}\left(\left|D_{0}(f)\right|\right)\right]^{p} \frac{1}{\left(1+\rho\left(x, y_{\tau}^{0, \nu}\right)\right)^{d+\epsilon}}\right)\right.\right. \\
& \left.\left.\times\left[\int_{X} \frac{1}{(1+\rho(x, z))^{d+\epsilon}} \mathrm{d} \mu(z)\right]^{p / p^{\prime}} \mathrm{d} \mu(x)\right]^{q / p}\right\}^{1 / q} \\
& \leqslant C\left\{\sum _ { l = 0 } ^ { \infty } 2 ^ { l ( s - \epsilon ) q } \left(\sum_{\tau \in I_{0}} \sum_{\nu=1}^{N(0, \tau)} \mu\left(Q_{\tau}^{0, \nu}\right)\left[m_{Q_{\tau}^{0, \nu}}\left(\left|D_{0}(f)\right|\right)\right]^{p}\right.\right. \\
& \left.\left.\times \int_{X} \frac{1}{\left(1+\rho\left(x, y_{\tau}^{0, \nu}\right)\right)^{d+\epsilon}} \mathrm{d} \mu(x)\right)^{q / p}\right\}^{1 / q} \\
& \leqslant C\left\{\sum_{\tau \in I_{0}} \sum_{\nu=1}^{N(0, \tau)} \mu\left(Q_{\tau}^{0, \nu}\right)\left[m_{Q_{\tau}^{0, \nu}}\left(\left|D_{0}(f)\right|\right)\right]^{p}\right\}^{1 / p} \\
& \leqslant C\|f\|_{B_{p q}^{s}(X)} .
\end{aligned}
$$

Thus, in all cases, we obtain

$$
E_{1} \leqslant C\|f\|_{B_{p q}^{s}(X)}
$$

which is the desired estimate for $E_{1}$.

The estimate for $E_{2}$ is similar. When $p \leqslant 1$, we use (3.9), the Hölder inequality, the regularity (ii) of $\tilde{D}_{k}$ and choose $\epsilon \in(s, \theta)$ such that $d<(d+\epsilon) p$ and $2^{-k}+\rho\left(x, y_{\tau}^{k, \nu}\right) \sim$ 
$2^{-k}+\rho(x, y)$ for any $y \in Q_{\tau}^{k, \nu}$. It follows that

$$
\begin{aligned}
& E_{2} \leqslant C\left\{\sum _ { l = 0 } ^ { \infty } 2 ^ { l s q } \left(2 ^ { l d } \int _ { X } \int _ { B ( x , 2 ^ { - l } ) } \left[\sum_{k=1}^{l+1} \sum_{\tau \in I_{k}} \sum_{\nu=1}^{N(k, \tau)} \mu\left(Q_{\tau}^{k, \nu}\right)\left|D_{k}(f)\left(y_{\tau}^{k, \nu}\right)\right|\right.\right.\right. \\
& \left.\left.\left.\times \frac{2^{-k \epsilon} \rho(x, y)^{\epsilon}}{\left(2^{-k}+\rho\left(x, y_{\tau}^{k, \nu}\right)\right)^{d+2 \epsilon}}\right]^{p} \mathrm{~d} \mu(y) \mathrm{d} \mu(x)\right)^{q / p}\right\}^{1 / q} \\
& \leqslant C\left\{\sum _ { l = 0 } ^ { \infty } 2 ^ { l ( s - \epsilon ) q } \left(\int _ { X } \left[\sum_{k=1}^{l+1} \sum_{\tau \in I_{k}} \sum_{\nu=1}^{N(k, \tau)} \mu\left(Q_{\tau}^{k, \nu}\right)\left|D_{k}(f)\left(y_{\tau}^{k, \nu}\right)\right|\right.\right.\right. \\
& \left.\left.\left.\times \frac{2^{-k \epsilon}}{\left(2^{-k}+\rho\left(x, y_{\tau}^{k, \nu}\right)\right)^{d+2 \epsilon}}\right]^{p} \mathrm{~d} \mu(x)\right)^{q / p}\right\}^{1 / q} \\
& \leqslant C\left\{\sum _ { l = 0 } ^ { \infty } 2 ^ { l ( s - \epsilon ) q } \left[\sum_{k=1}^{l+1} \sum_{\tau \in I_{k}} \sum_{\nu=1}^{N(k, \tau)} \mu\left(Q_{\tau}^{k, \nu}\right)^{p}\left|D_{k}(f)\left(y_{\tau}^{k, \nu}\right)\right|^{p}\right.\right. \\
& \left.\left.\times \int_{X} \frac{1}{\left(2^{-k}+\rho\left(x, y_{\tau}^{k, \nu}\right)\right)^{(d+\epsilon) p}} \mathrm{~d} \mu(x)\right]^{q / p}\right\}^{1 / q} \\
& \leqslant C\left\{\sum_{l=0}^{\infty} 2^{l(s-\epsilon) q}\left[\sum_{k=1}^{l+1} 2^{k \epsilon p} \sum_{\tau \in I_{k}} \sum_{\nu=1}^{N(k, \tau)} \mu\left(Q_{\tau}^{k, \nu}\right)\left|D_{k}(f)\left(y_{\tau}^{k, \nu}\right)\right|^{p}\right]^{q / p}\right\}^{1 / q} \\
& \leqslant C\left\{\sum_{l=0}^{\infty}\left[\sum_{k=1}^{l+1} 2^{(k-l)(\epsilon-s) p} 2^{k s p}\left\|D_{k}(f)\right\|_{L^{p}(X)}^{p}\right]^{q / p}\right\}^{1 / q} \\
& \leqslant \begin{cases}C\left\{\sum_{l=0}^{\infty} \sum_{k=1}^{l+1} 2^{(k-l)(\epsilon-s) q} 2^{k s q}\left\|D_{k}(f)\right\|_{L^{p}(X)}^{q}\right\}^{1 / q}, & 0<q / p \leqslant 1, \\
C\left\{\sum_{l=0}^{\infty}\left[\sum_{k=1}^{l+1} 2^{(k-l)(\epsilon-s) q / 2} 2^{k s q}\left\|D_{k}(f)\right\|_{L^{p}(X)}^{q}\right]\left[\sum_{k=1}^{l+1} 2^{(k-l)(\epsilon-s) p(q / p)^{\prime}}\right]^{1-p / q}\right\}^{1 / q}, & 1<q / p \leqslant \infty,\end{cases} \\
& \leqslant C\left\{\sum_{k=1}^{\infty} 2^{k s q}\left\|D_{k}(f)\right\|_{L^{p}(X)}^{q}\right\}^{1 / q} \\
& \leqslant C\|f\|_{B_{p q}^{s}(X)} \text {. }
\end{aligned}
$$

If $p>1$, we may use similar computations, which we omit, to see that

$$
\begin{aligned}
E_{2} \leqslant C\left\{\sum _ { l = 0 } ^ { \infty } 2 ^ { l ( s - \epsilon ) q } \left(\int _ { X } \left[\sum_{k=1}^{l+1} \sum_{\tau \in I_{k}} \sum_{\nu=1}^{N(k, \tau)} \mu\left(Q_{\tau}^{k, \nu}\right)\left|D_{k}(f)\left(y_{\tau}^{k, \nu}\right)\right|\right.\right.\right. \\
\left.\left.\left.\times \frac{2^{-k \epsilon}}{\left(2^{-k}+\rho\left(x, y_{\tau}^{k, \nu}\right)\right)^{d+2 \epsilon}}\right]^{p} \mathrm{~d} \mu(x)\right)^{q / p}\right\}^{1 / q}
\end{aligned}
$$




$$
\begin{aligned}
& \leqslant C\left\{\sum _ { l = 0 } ^ { \infty } 2 ^ { l s q } \left(\int _ { X } \left[\sum_{k=1}^{l+1} 2^{(k-l) \epsilon_{1} p} \sum_{\tau \in I_{k}} \sum_{\nu=1}^{N(k, \tau)} \mu\left(Q_{\tau}^{k, \nu}\right)\left|D_{k}(f)\left(y_{\tau}^{k, \nu}\right)\right|^{p}\right.\right.\right. \\
& \left.\times \frac{2^{-k \epsilon}}{\left(2^{-k}+\rho\left(x, y_{\tau}^{k, \nu}\right)\right)^{d+\epsilon}}\right] \\
& \left.\left.\times\left[\sum_{k=1}^{l+1} 2^{(k-l) \epsilon_{2} p^{\prime}} \int_{X} \frac{2^{-k \epsilon}}{\left(2^{-k}+\rho(x, z)\right)^{d+\epsilon}} \mathrm{d} \mu(z)\right]^{p / p^{\prime}} \mathrm{d} \mu(x)\right)^{q / p}\right\}^{1 / q} \\
& \leqslant C\left\{\sum_{l=0}^{\infty}\left[\sum_{k=1}^{l+1} 2^{(k-l)\left(\epsilon_{1}-s\right) p} 2^{k s p} \sum_{\tau \in I_{k}} \sum_{\nu=1}^{N(k, \tau)} \mu\left(Q_{\tau}^{k, \nu}\right)\left|D_{k}(f)\left(y_{\tau}^{k, \nu}\right)\right|^{p}\right]^{q / p}\right\}^{1 / q} \\
& \leqslant C\left\{\sum_{l=0}^{\infty}\left[\sum_{k=1}^{l+1} 2^{(k-l)\left(\epsilon_{1}-s\right) p} 2^{k s p}\left\|D_{k}(f)\right\|_{L^{p}(X)}^{p}\right]^{q / p}\right\}^{1 / q} \\
& \leqslant C\|f\|_{B_{p q}^{s}(X) .}
\end{aligned}
$$

Here, we have chosen $\epsilon_{1}>0$ and $\epsilon_{2}>0$ such that $\epsilon=\epsilon_{1}+\epsilon_{2}$ and $\epsilon_{1}>s$. Again, we obtain in both cases

$$
E_{2} \leqslant C\|f\|_{B_{p q}^{s}(X)}
$$

which is the desired estimate for $E_{2}$.

Finally, let us estimate $E_{3}$. Decompose the estimate for $E_{3}$ as follows:

$$
\begin{aligned}
E_{3} \leqslant & C\left\{\sum _ { l = 0 } ^ { \infty } 2 ^ { l s q } \left[2^{l d} \int_{X} \int_{B\left(x, 2^{-l}\right)} \mid \sum_{k=l+2}^{\infty} \sum_{\tau \in I_{k}} \sum_{\nu=1}^{N(k, \tau)} \mu\left(Q_{\tau}^{k, \nu}\right) D_{k}(f)\left(y_{\tau}^{k, \nu}\right)\right.\right. \\
& \left.\left.\times\left.\tilde{D}_{k}\left(x, y_{\tau}^{k, \nu}\right)\right|^{p} \mathrm{~d} \mu(y) \mathrm{d} \mu(x)\right]^{q / p}\right\}^{1 / q} \\
+ & C\left\{\sum _ { l = 0 } ^ { \infty } 2 ^ { l s q } \left[2^{l d} \int_{X} \int_{B\left(x, 2^{-l}\right)} \mid \sum_{k=l+2}^{\infty} \sum_{\tau \in I_{k}} \sum_{\nu=1}^{N(k, \tau)} \mu\left(Q_{\tau}^{k, \nu}\right) D_{k}(f)\left(y_{\tau}^{k, \nu}\right)\right.\right. \\
& \left.\left.\times\left.\tilde{D}_{k}\left(y, y_{\tau}^{k, \nu}\right)\right|^{p} \mathrm{~d} \mu(y) \mathrm{d} \mu(x)\right]^{q / p}\right\}^{1 / q} \\
=E_{3}^{1}+E_{3}^{2} . &
\end{aligned}
$$

When $p \leqslant 1$, we use the size condition (i) of $\tilde{D}_{k}$ in Lemma 3.6 with a choice of $\epsilon \in(0, \theta)$ such that $d<(d+\epsilon) p,(3.9)$, together with Hölder's inequality and the fact that

$$
2^{-l}+\rho\left(x, y_{\tau}^{k, \nu}\right) \sim 2^{-l}+\rho(x, z)
$$

for all $z \in Q_{\tau}^{k, \nu}$ and all $x \in X, s>0$ and the arbitrariness of $y_{\tau}^{k, \nu}$. We can hence estimate $E_{3}^{1}$ by

$$
E_{3}^{1} \leqslant C\left\{\sum_{l=0}^{\infty} 2^{l s q}\left[\int_{X}\left|\sum_{k=l+2}^{\infty} \sum_{\tau \in I_{k}} \sum_{\nu=1}^{N(k, \tau)} \mu\left(Q_{\tau}^{k, \nu}\right) D_{k}(f)\left(y_{\tau}^{k, \nu}\right) \tilde{D}_{k}\left(x, y_{\tau}^{k, \nu}\right)\right|^{p} \mathrm{~d} \mu(x)\right]^{q / p}\right\}^{1 / q}
$$




$$
\begin{aligned}
& \leqslant C\left\{\sum _ { l = 0 } ^ { \infty } \left(\int _ { X } \left[\sum_{k=l+2}^{\infty} \sum_{\tau \in I_{k}} \sum_{\nu=1}^{N(k, \tau)} 2^{(l-k) s} 2^{k s} \mu\left(Q_{\tau}^{k, \nu}\right)\left|D_{k}(f)\left(y_{\tau}^{k, \nu}\right)\right|\right.\right.\right. \\
& \left.\left.\left.\times \frac{2^{-k \epsilon}}{\left(2^{-k}+\rho\left(x, y_{\tau}^{k, \nu}\right)\right)^{d+\epsilon}}\right]^{p} \mathrm{~d} \mu(x)\right)^{q / p}\right\}^{1 / q} \\
& \leqslant C\left\{\sum _ { l = 0 } ^ { \infty } \left[\sum_{k=l+2}^{\infty} \sum_{\tau \in I_{k}} \sum_{\nu=1}^{N(k, \tau)} 2^{(l-k) s p} 2^{k s p} \mu\left(Q_{\tau}^{k, \nu}\right)\left|D_{k}(f)\left(y_{\tau}^{k, \nu}\right)\right|^{p}\right.\right. \\
& \left.\left.\times 2^{k d(1-p)} \int_{X} \frac{2^{-k \epsilon p}}{\left(2^{-k}+\rho\left(x, y_{\tau}^{k, \nu}\right)\right)^{(d+\epsilon) p}} \mathrm{~d} \mu(x)\right]^{q / p}\right\}^{1 / q} \\
& \leqslant C\left\{\sum_{l=0}^{\infty}\left[\sum_{k=l+2}^{\infty} \sum_{\tau \in I_{k}} \sum_{\nu=1}^{N(k, \tau)} 2^{(l-k) s p} 2^{k s p} \mu\left(Q_{\tau}^{k, \nu}\right)\left|D_{k}(f)\left(y_{\tau}^{k, \nu}\right)\right|^{p}\right]^{q / p}\right\}^{1 / q} \\
& \leqslant C\left\{\sum_{l=0}^{\infty}\left[\sum_{k=l+2}^{\infty} 2^{(l-k) s} 2^{k s p}\left\|D_{k}(f)\right\|_{L^{p}(X)}^{p}\right]^{q / p}\right\}^{1 / q} \\
& \leqslant\left\{\begin{array}{c}
C\left\{\sum_{l=0}^{\infty} \sum_{k=l+2}^{\infty} 2^{(l-k) s q / p} 2^{k s q}\left\|D_{k}(f)\right\|_{L^{p}(X)}^{q}\right\}^{1 / q}, \quad 0<q / p \leqslant 1 \\
C\left\{\sum_{l=0}^{\infty} \sum_{k=l+2}^{\infty} 2^{(l-k) s q /(2 p)} 2^{k s q}\left\|D_{k}(f)\right\|_{L^{p}(X)}^{q}\left(\sum_{k=l+2}^{\infty} 2^{(l-k) s(q / p)^{\prime} / 2}\right)^{1-p / q}\right\}^{1 / q} \\
1<q / p \leqslant \infty
\end{array}\right. \\
& \leqslant C\left\{\sum_{k=2}^{\infty} 2^{k s q}\left\|D_{k}(f)\right\|_{L^{p}(X)}^{q}\right\}^{1 / q} \\
& \leqslant C\|f\|_{B_{p q}^{s}(X)} .
\end{aligned}
$$

If $p>1$, we have

$$
\begin{aligned}
E_{3}^{1} \leqslant C\left\{\sum _ { l = 0 } ^ { \infty } \left(\int _ { X } \left[\sum_{k=l+2}^{\infty} \sum_{\tau \in I_{k}} \sum_{\nu=1}^{N(k, \tau)} 2^{(l-k) s} 2^{k s} \mu\left(Q_{\tau}^{k, \nu}\right)\left|D_{k}(f)\left(y_{\tau}^{k, \nu}\right)\right|\right.\right.\right. \\
\left.\left.\left.\times \frac{2^{-k \epsilon}}{\left(2^{-k}+\rho\left(x, y_{\tau}^{k, \nu}\right)\right)^{d+\epsilon}}\right]^{p} \mathrm{~d} \mu(x)\right)^{q / p}\right\}^{1 / q} \\
\leqslant C\left\{\sum _ { l = 0 } ^ { \infty } \left(\int _ { X } \left[\sum_{k=l+2}^{\infty} \sum_{\tau \in I_{k}} \sum_{\nu=1}^{N(k, \tau)} 2^{(l-k) s} 2^{k s p} \mu\left(Q_{\tau}^{k, \nu}\right)\left|D_{k}(f)\left(y_{\tau}^{k, \nu}\right)\right|^{p}\right.\right.\right. \\
\left.\left.\times\left[\sum_{k=l+2}^{\infty} 2^{(l-k) s} \int_{X} \frac{2^{-k \epsilon}}{\left(2^{-k}+\rho\left(x, y_{\tau}^{k, \nu}\right)\right)^{d+\epsilon}}\right]^{q / p} \mathrm{~d} \mu(z, z)\right)^{d+p^{\prime}} \mathrm{d} \mu(x)\right)^{1 / q}
\end{aligned}
$$




$$
\begin{aligned}
& \leqslant C\left\{\sum _ { l = 0 } ^ { \infty } \left[\sum_{k=l+2}^{\infty} 2^{(l-k) s} 2^{k s p} \sum_{\tau \in I_{k}} \sum_{\nu=1}^{N(k, \tau)} \mu\left(Q_{\tau}^{k, \nu}\right)\left|D_{k}(f)\left(y_{\tau}^{k, \nu}\right)\right|^{p}\right.\right. \\
& \left.\left.\times \int_{X} \frac{2^{-k \epsilon}}{\left(2^{-k}+\rho\left(x, y_{\tau}^{k, \nu}\right)\right)^{d+\epsilon}} \mathrm{d} \mu(x)\right]^{q / p}\right\}^{1 / q} \\
& \leqslant C\left\{\sum_{l=0}^{\infty}\left[\sum_{k=l+2}^{\infty} 2^{(l-k) s} 2^{k s p}\left\|D_{k}(f)\right\|_{L^{p}(X)}^{p}\right]^{q / p}\right\}^{1 / q} \\
& \leqslant C\|f\|_{B_{p q}^{s}(X) .}
\end{aligned}
$$

We have omitted some similar computations as for the case $p \leqslant 1$. Thus, we always have

$$
E_{3}^{1} \leqslant C\|f\|_{B_{p q}^{s}(X)}
$$

which is the desired estimate.

Similar to the estimate for (3.14), using analogous estimates for $E_{3}^{2}$, we have

$$
\begin{gathered}
E_{3}^{2} \leqslant C\left\{\sum _ { l = 0 } ^ { \infty } 2 ^ { l s q } \left(2 ^ { l d } \int _ { X } \int _ { B ( x , 2 ^ { - l } ) } \left[\sum_{k=l+2}^{\infty} \sum_{\tau \in I_{k}} \sum_{\nu=1}^{N(k, \tau)} \mu\left(Q_{\tau}^{k, \nu}\right)\left|D_{k}(f)\left(y_{\tau}^{k, \nu}\right)\right|\right.\right.\right. \\
\left.\left.\left.\times \frac{2^{-k \epsilon}}{\left(2^{-k}+\rho\left(y, y_{\tau}^{k, \nu}\right)\right)^{d+\epsilon}}\right]^{p} \mathrm{~d} \mu(y) \mathrm{d} \mu(x)\right)^{q / p}\right\}^{1 / q} \\
\leqslant C\left\{\sum _ { l = 0 } ^ { \infty } \left(\int _ { X } \left[\sum_{k=l+2}^{\infty} \sum_{\tau \in I_{k}} \sum_{\nu=1}^{N(k, \tau)} 2^{(l-k) s} 2^{k s} \mu\left(Q_{\tau}^{k, \nu}\right)\left|D_{k}(f)\left(y_{\tau}^{k, \nu}\right)\right|\right.\right.\right. \\
\times C\|f\|_{B_{p q}^{s}(X) .}
\end{gathered}
$$

From (3.14) and (3.15), it follows that

$$
E_{3} \leqslant C\|f\|_{B_{p q}^{s}(X)}
$$

Estimates (3.12), (3.13) and (3.16) tell us that

$$
\left\{\sum_{l=0}^{\infty} 2^{l s q}\left(2^{l d} \int_{X} \int_{B\left(x, 2^{-l}\right)}|f(x)-f(y)|^{p} \mathrm{~d} \mu(y) \mathrm{d} \mu(x)\right)^{q / p}\right\}^{1 / q} \leqslant C\|f\|_{B_{p q}^{s}(X)} .
$$

Combining estimates (3.7), (3.10), (3.11) and (3.17) with Definition 2.1, we see that

$$
\|f\|_{L(s, p, q, X)} \leqslant C\|f\|_{B_{p q}^{s}(X)} .
$$

This proves (i), which completes the proof of Proposition 3.7. 
Combining Proposition 3.5 with Proposition 3.7, we obtain the following theorem.

Theorem 3.8. If $0<s<\theta, 1 \leqslant p \leqslant \infty$ and $0<q \leqslant \infty$, then

$$
L(s, p, q, X)=L_{\mathrm{b}}(s, p, q, X)=B_{p q}^{s}(X)
$$

with equivalence of norms.

We remark that if $X=\mathbb{R}^{n}$, the restriction on the indices of the spaces $L(s, p, q, X)$, $L_{\mathrm{b}}(s, p, q, X)$ and $B_{p q}^{s}(X)$ in Theorem 3.8 is exactly the same as that of Theorem 3.5.1 in [45]. Even when $X=\mathbb{R}^{n}$, it is still not clear if Theorem 3.8 is true when $p<1$.

Also, as mentioned above, our new Lipschitz-type spaces, $L_{\mathrm{t}}(s, p, q, X)$, are related to Triebel-Lizorkin spaces on spaces of homogeneous type, as we will show in Theorem 3.12 below. To see this, let us first establish the following proposition.

Proposition 3.9. If $0<s<\theta, 1 \leqslant p<\infty$ and $0<q \leqslant \infty$, then

$$
L_{\mathrm{t}}(s, p, q, X) \subset F_{p q}^{s}(X) .
$$

Moreover, there is a constant $C>0$ such that for all $f \in L_{\mathrm{t}}(s, p, q, X)$,

$$
\|f\|_{F_{p q}^{s}(X)} \leqslant C\|f\|_{L_{\mathrm{t}}(s, p, q, X)} .
$$

Proof. Let $f \in L_{\mathrm{t}}(s, p, q, X)$. Then inequality (3.2) in the proof of Proposition 3.5 still holds in this case. By Hölder's inequality, the support condition, the size condition and the vanishing moment property of $D_{k}$, we have

$$
\begin{aligned}
\left\|\left\{\sum_{k=1}^{\infty}\left[2^{k s}\left|D_{k}(f)\right|\right]^{q}\right\}^{1 / q}\right\|_{L^{p}(X)} & \\
& =\left[\int_{X}\left\{\sum_{k=1}^{\infty} 2^{k s q}\left|\int_{X} D_{k}(x, y)[f(y)-f(x)] \mathrm{d} \mu(y)\right|^{q}\right\}^{p / q} \mathrm{~d} \mu(x)\right]^{1 / p} \\
& \leqslant C\left\{\int_{X}\left[\sum_{k=1}^{\infty} 2^{k s q}\left(2^{k d} \int_{B\left(\cdot, C 2^{-k}\right)}|f(y)-f(x)| \mathrm{d} \mu(y)\right)^{q}\right]^{p / q} \mathrm{~d} \mu(x)\right\}^{1 / p} \\
& \leqslant C\|f\|_{L_{\mathrm{t}}(s, p, q, X)} .
\end{aligned}
$$

Estimates (3.3) and (3.18) and Definition 3.4 imply that

$$
\|f\|_{F_{p q}^{s}(X)} \leqslant C\|f\|_{L_{\mathrm{t}}(s, p, q, X)},
$$

where $C$ is independent of $f$. That is, we have $L_{\mathrm{t}}(s, p, q, X) \subset F_{p q}^{s}(X)$.

This finishes the proof of Proposition 3.9.

To establish the converse of Proposition 3.9, we first recall the following well-known lemma which can be found in $\left[\mathbf{1 2}\right.$, pp. 147, 148] for $\mathbb{R}^{n}$ and $[\mathbf{2 2}$, p. 93] for spaces of homogeneous type. 
Lemma 3.10. Let $0<r \leqslant 1, k, \eta \in \mathbb{Z}$ with $\eta \leqslant k$. Suppose that for any dyadic cube $Q_{\tau}^{k, \nu}$,

$$
\left|f_{Q_{\tau}^{k, \nu}}(x)\right| \leqslant\left(1+2^{\eta} \rho\left(x, y_{\tau}^{k, \nu}\right)\right)^{-d-\gamma}
$$

where $y_{\tau}^{k, \nu}$ is any point in $Q_{\tau}^{k, \nu}$ and $\gamma>d(1 / r-1)$. Then for all $x \in X$,

$$
\sum_{\tau \in I_{k}} \sum_{\nu=1}^{N(k, \tau)}\left|\lambda_{Q_{\tau}^{k, \nu}}\right|\left|f_{Q_{\tau}^{k, \nu}}(x)\right| \leqslant C 2^{(k-\eta) d / r}\left[M\left(\sum_{\tau \in I_{k}} \sum_{\nu=1}^{N(k, \tau)}\left|\lambda_{Q_{\tau}^{k, \nu}}\right|^{r} \chi_{Q_{\tau}^{k, \nu}}\right)(x)\right]^{1 / r}
$$

where $C$ is independent of $x, k$ and $\eta$, and $M$ is the Hardy-Littlewood maximal operator on $X$.

Proposition 3.11. If $0<s<\theta, 1<p<\infty$ and $1<q \leqslant \infty$, then

$$
F_{p q}^{s}(X) \subset L_{\mathrm{t}}(s, p, q, X) .
$$

Moreover, there exists a constant $C>0$ such that for all $f \in F_{p q}^{s}(X)$,

$$
\|f\|_{L_{\mathrm{t}}(s, p, q, X)} \leqslant C\|f\|_{B_{p q}^{s}(X)} .
$$

Proof. Let $1<p<\infty$. Similarly to (3.5) and (3.6), we have

$$
F_{p q}^{s}(X) \subset F_{p 2}^{0}(X)=L^{p}(X) .
$$

Thus, we obtain

$$
\|f\|_{L^{p}(X)} \leqslant C\|f\|_{F_{p q}^{s}(X)} .
$$

We now estimate the second term appearing in the definition of the norm of the space $L_{\mathrm{t}}(s, p, q, X)$. To do so, without loss of generality, we may assume that $C_{3}=1$. Expressing $f$ as in (3.4), we have

$$
\begin{aligned}
& \left\|\left\{\sum_{l=0}^{\infty} 2^{l s q}\left[2^{l d} \int_{B\left(\cdot, 2^{-l}\right)}|f(y)-f(\cdot)| \mathrm{d} \mu(y)\right]^{q}\right\}^{1 / q}\right\|_{L^{p}(X)} \\
& \leqslant \|\left\{\sum _ { l = 0 } ^ { \infty } 2 ^ { l s q } \left(2 ^ { l d } \int _ { B ( \cdot , 2 ^ { - l } ) } \left[\sum_{\tau \in I_{0}} \sum_{\nu=1}^{N(0, \tau)} \mu\left(Q_{\tau}^{0, \nu}\right) m_{Q_{\tau}^{0, \nu}}\left(\left|D_{0}(f)\right|\right)\right.\right.\right.
\end{aligned}
$$

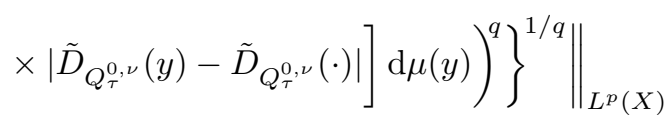

$$
\begin{aligned}
& +\|\left\{\sum _ { l = 0 } ^ { \infty } 2 ^ { l s q } \left(2 ^ { l d } \int _ { B ( \cdot , 2 ^ { - l } ) } \left[\sum_{k=1}^{l+1} \sum_{\tau \in I_{k}} \sum_{\nu=1}^{N(k, \tau)} \mu\left(Q_{\tau}^{k, \nu}\right)\left|D_{k}(f)\left(y_{\tau}^{k, \nu}\right)\right|\right.\right.\right. \\
& \left.\left.\left.\times\left|\tilde{D}_{k}\left(y, y_{\tau}^{k, \nu}\right)-\tilde{D}_{k}\left(\cdot, y_{\tau}^{k, \nu}\right)\right|\right] \mathrm{d} \mu(y)\right)^{q}\right\}^{1 / q} \|_{L^{p}(X)}
\end{aligned}
$$


734

D. Yang and Y. Lin

$$
\begin{aligned}
& +\|\left\{\sum _ { l = 0 } ^ { \infty } 2 ^ { l s q } \left(2 ^ { l d } \int _ { B ( \cdot , 2 ^ { - l } ) } \left[\sum_{k=l+2}^{\infty} \sum_{\tau \in I_{k}} \sum_{\nu=1}^{N(k, \tau)} \mu\left(Q_{\tau}^{k, \nu}\right)\left|D_{k}(f)\left(y_{\tau}^{k, \nu}\right)\right|\right.\right.\right. \\
& \left.\left.\left.\quad \times\left|\tilde{D}_{k}\left(y, y_{\tau}^{k, \nu}\right)-\tilde{D}_{k}\left(\cdot, y_{\tau}^{k, \nu}\right)\right|\right] \mathrm{d} \mu(y)\right)^{q}\right\}^{1 / q} \|_{L^{p}(X)} \\
& =G_{1}+G_{2}+G_{3} .
\end{aligned}
$$

When $p / q \leqslant 1$, we may use Lemma 3.3, the regularity of $\tilde{D}_{Q_{\tau}^{0, \nu}}$ from Lemma 3.6 (iv), together with the fact that $\epsilon \in(s, \theta)$, Hölder's inequality, $(3.9)$, the fact that $y_{\tau}^{k, \nu}$ is arbitrary in $Q_{\tau}^{k, \nu}$ and that $1+\rho\left(x, y_{\tau}^{0, \nu}\right) \sim 1+\rho(x, z)$ for any $z \in Q_{\tau}^{0, \nu}, \mu\left(Q_{\tau}^{0, \nu}\right) \sim C$. It follows that

$$
\begin{aligned}
& G_{1} \leqslant C\left[\int _ { X } \left\{\sum _ { l = 0 } ^ { \infty } 2 ^ { l s q } \left(2 ^ { l d } \int _ { B ( x , 2 ^ { - l } ) } \left[\sum_{\tau \in I_{0}} \sum_{\nu=1}^{N(0, \tau)} \mu\left(Q_{\tau}^{0, \nu}\right) m_{Q_{\tau}^{0, \nu}}\left(\left|D_{0}(f)\right|\right)\right.\right.\right.\right. \\
& \left.\left.\left.\left.\times \frac{\rho(x, y)^{\epsilon}}{\left(1+\rho\left(x, y_{\tau}^{0, \nu}\right)\right)^{d+2 \epsilon}}\right] \mathrm{d} \mu(y)\right)^{q}\right\}^{p / q} \mathrm{~d} \mu(x)\right]^{1 / p} \\
& \leqslant C\left[\int _ { X } \left\{\sum _ { l = 0 } ^ { \infty } 2 ^ { l ( s - \epsilon ) q } \left[\sum_{\tau \in I_{0}} \sum_{\nu=1}^{N(0, \tau)} \mu\left(Q_{\tau}^{0, \nu}\right) m_{Q_{\tau^{0}}^{0, \nu}}\left(\left|D_{0}(f)\right|\right)\right.\right.\right. \\
& \left.\left.\left.\times \frac{1}{\left(1+\rho\left(x, y_{\tau}^{0, \nu}\right)\right)^{d+\epsilon}}\right]^{q}\right\}^{p / q} \mathrm{~d} \mu(x)\right]^{1 / p} \\
& \leqslant C\left[\int _ { X } \left\{\sum_{l=0}^{\infty} 2^{l(s-\epsilon) q}\left(\sum_{\tau \in I_{0}} \sum_{\nu=1}^{N(0, \tau)} \mu\left(Q_{\tau}^{0, \nu}\right)\left[m_{Q_{\tau}^{0, \nu}}\left(\left|D_{0}(f)\right|\right)\right]^{p} \frac{1}{\left(1+\rho\left(x, y_{\tau}^{0, \nu}\right)\right)^{d+\epsilon}}\right)^{q / p}\right.\right. \\
& \left.\left.\times\left[\int_{X} \frac{1}{(1+\rho(x, z))^{d+\epsilon}} \mathrm{d} \mu(z)\right]^{q / p^{\prime}}\right\}^{p / q} \mathrm{~d} \mu(x)\right]^{1 / p} \\
& \leqslant C\left[\int _ { X } \left\{\sum _ { l = 0 } ^ { \infty } 2 ^ { l ( s - \epsilon ) q } \left(\sum_{\tau \in I_{0}} \sum_{\nu=1}^{N(0, \tau)} \mu\left(Q_{\tau}^{0, \nu}\right)\left[m_{Q_{\tau}^{0, \nu}}\left(\left|D_{0}(f)\right|\right)\right]^{p}\right.\right.\right. \\
& \left.\left.\left.\times \frac{1}{\left(1+\rho\left(x, y_{\tau}^{0, \nu}\right)\right)^{d+\epsilon}}\right)^{q / p}\right\}^{p / q} \mathrm{~d} \mu(x)\right]^{1 / p} \\
& \leqslant C\left\{\int _ { X } \sum _ { l = 0 } ^ { \infty } 2 ^ { l ( s - \epsilon ) p } \left(\sum_{\tau \in I_{0}} \sum_{\nu=1}^{N(0, \tau)} \mu\left(Q_{\tau}^{0, \nu}\right)\left[m_{Q_{\tau}^{0, \nu}}\left(\left|D_{0}(f)\right|\right)\right]^{p}\right.\right. \\
& \left.\left.\times \frac{1}{\left(1+\rho\left(x, y_{\tau}^{0, \nu}\right)\right)^{d+\epsilon}}\right) \mathrm{d} \mu(x)\right\}^{1 / p} \\
& \leqslant C\left\{\sum_{l=0}^{\infty} 2^{l(s-\epsilon) p} \sum_{\tau \in I_{0}} \sum_{\nu=1}^{N(0, \tau)} \mu\left(Q_{\tau}^{0, \nu}\right)\left[m_{Q_{\tau}^{0, \nu}}\left(\left|D_{0}(f)\right|\right)\right]^{p}\right. \\
& \left.\times \int_{X} \frac{1}{\left(1+\rho\left(x, y_{\tau}^{0, \nu}\right)\right)^{d+\epsilon}} \mathrm{d} \mu(x)\right\}^{1 / p}
\end{aligned}
$$

https://doi.org/10.1017/S0013091503000907 Published online by Cambridge University Press 


$$
\begin{aligned}
& \leqslant C\left\{\sum_{l=0}^{\infty} 2^{l(s-\epsilon) p}\right\}^{1 / p}\left\{\sum_{\tau \in I_{0}} \sum_{\nu=1}^{N(0, \tau)} \mu\left(Q_{\tau}^{0, \nu}\right)\left[m_{Q_{\tau}^{0, \nu}}\left(\left|D_{0}(f)\right|\right)\right]^{p}\right\}^{1 / p} \\
& \leqslant C\left\{\sum_{\tau \in I_{0}} \sum_{\nu=1}^{N(0, \tau)} \mu\left(Q_{\tau}^{0, \nu}\right)\left[m_{Q_{\tau}^{0, \nu}}\left(\left|D_{0}(f)\right|\right)\right]^{p}\right\}^{1 / p} \\
& \leqslant C\|f\|_{F_{p q}^{s}(X)} .
\end{aligned}
$$

When $p / q>1$, similar arguments yield that

$$
\begin{aligned}
G_{1} & \leqslant C\left[\int _ { X } \left\{\sum _ { l = 0 } ^ { \infty } 2 ^ { l ( s - \epsilon ) q } \left(\sum_{\tau \in I_{0}} \sum_{\nu=1}^{N(0, \tau)} \mu\left(Q_{\tau}^{0, \nu}\right)\left[m_{Q_{\tau}^{0, \nu}}\left(\left|D_{0}(f)\right|\right)\right]^{p}\right.\right.\right. \\
& \left.\leqslant\left[\int_{X}\left\{\sum_{l=0}^{\infty} 2^{l(s-\epsilon) q}\left(\sum_{\tau \in I_{0}} \sum_{\nu=1}^{N(0, \tau)} \mu\left(Q_{\tau}^{0, \nu}\right)\left[m_{Q_{\tau}^{0, \nu}}\left(\left|D_{0}(f)\right|\right)\right]^{p} \frac{1}{\left(1+\rho\left(x, y_{\tau}^{0, \nu}\right)\right)^{d+\epsilon}}\right)\right\}^{q / p}\right\}^{p / q} \mathrm{~d} \mu(x)\right]^{1 / p} \\
& \leqslant C\left\{\sum_{l=0}^{\infty} 2^{l(s-\epsilon) q} \sum_{\tau \in I_{0}} \sum_{\nu=1}^{N(0, \tau)} \mu\left(\sum_{l=0}^{\infty} 2^{l(s-\epsilon) q}\right\}^{p / q-1} \mathrm{~d} \mu(x)\right]^{1 / p} \\
& \leqslant C\left\{\sum_{l=0}^{\infty} 2^{l(s-\epsilon) q}\right\}^{1 / p}\left\{\sum_{\tau \in I_{0}} \sum_{\nu=1}^{N(0, \tau)} \mu\left(m_{Q_{\tau}^{0, \nu}}\left(\left|D_{0}(f)\right|\right)\right]^{p}\right. \\
\leqslant & \leqslant C\left\{\sum_{\tau \in I_{0}} \sum_{\nu=1}^{N(0, \tau)} \mu\left(Q_{\tau}^{0, \nu}\right)\left[m_{Q_{\tau}^{0, \nu}}\left(\left|D_{0}(f)\right|\right)\right]^{p}\right\}^{1 / p} \\
\leqslant & \left.\leqslant C f \|_{F_{p q}^{s}(X) \cdot} \frac{1}{\left(1+\rho\left(x, y_{\tau}^{0, \nu}\right)\right)^{d+\epsilon}} \mathrm{d} \mu(x)\right\}^{1 / p}
\end{aligned}
$$

Thus, in both cases, we have

$$
G_{1} \leqslant C\|f\|_{F_{p q}^{s}(X)}
$$

which is the required estimate for $G_{1}$.

The regularity of $\tilde{D}_{k}$, Lemma 3.10 and the Fefferman-Stein vector-valued inequality [10] tell us that

$$
\begin{aligned}
G_{2} \leqslant C\left[\int _ { X } \left\{\sum _ { l = 0 } ^ { \infty } 2 ^ { l s q } \left(2^{l d} \int_{B\left(x, 2^{-l}\right)}\right.\right.\right. & {\left[\sum_{k=1}^{l+1} \sum_{\tau \in I_{k}} \sum_{\nu=1}^{N(k, \tau)} \mu\left(Q_{\tau}^{k, \nu}\right)\left|D_{k}(f)\left(y_{\tau}^{k, \nu}\right)\right|\right.} \\
& \left.\left.\left.\left.\times \frac{2^{-k \epsilon} \rho(x, y)^{\epsilon}}{\left(2^{-k}+\rho\left(x, y_{\tau}^{k, \nu}\right)\right)^{d+2 \epsilon}}\right] \mathrm{d} \mu(y)\right)^{q}\right\}^{p / q} \mathrm{~d} \mu(x)\right]^{1 / p}
\end{aligned}
$$




$$
\begin{aligned}
& \leqslant C\left[\int _ { X } \left\{\sum _ { l = 0 } ^ { \infty } \left[\sum_{k=1}^{l+1} 2^{(k-l)(\epsilon-s)} \sum_{\tau \in I_{k}} \sum_{\nu=1}^{N(k, \tau)} 2^{k s} \mu\left(Q_{\tau}^{k, \nu}\right)\left|D_{k}(f)\left(y_{\tau}^{k, \nu}\right)\right|\right.\right.\right. \\
& \left.\left.\left.\times \frac{2^{-k \epsilon}}{\left(2^{-k}+\rho\left(x, y_{\tau}^{k, \nu}\right)\right)^{d+\epsilon}}\right]^{q}\right\}^{p / q} \mathrm{~d} \mu(x)\right]^{1 / p} \\
& \leqslant C\left[\int _ { X } \left\{\sum _ { l = 0 } ^ { \infty } \left(\sum_{k=1}^{l+1} 2^{(k-l)(\epsilon-s)}\right.\right.\right. \\
& \left.\left.\left.\times\left[M\left(\sum_{\tau \in I_{k}} \sum_{\nu=1}^{N(k, \tau)} 2^{k s r}\left|D_{k}(f)\left(y_{\tau}^{k, \nu}\right)\right|^{r} \chi_{Q_{\tau}^{k, \nu}}\right)(x)\right]^{1 / r}\right)^{q}\right\}^{p / q} \mathrm{~d} \mu(x)\right]^{1 / p} \\
& \leqslant C\left[\int _ { X } \left\{\sum_{l=0}^{\infty}\left(\sum_{k=1}^{l+1} 2^{(k-l)(\epsilon-s)}\left[M\left(\sum_{\tau \in I_{k}} \sum_{\nu=1}^{N(k, \tau)} 2^{k s r}\left|D_{k}(f)\left(y_{\tau}^{k, \nu}\right)\right|^{r} \chi_{Q_{\tau}^{k, \nu}}\right)(x)\right]^{q / r}\right)\right.\right. \\
& \left.\left.\times\left[\sum_{k=1}^{l+1} 2^{(k-l)(\epsilon-s)}\right]^{q / q^{\prime}}\right\}^{p / q} \mathrm{~d} \mu(x)\right]^{1 / p} \\
& \leqslant C\left[\int _ { X } \left\{\sum_{k=1}^{\infty}\left(\sum_{l=k-1}^{\infty} 2^{(k-l)(\epsilon-s)}\right)\right.\right. \\
& \left.\left.\times\left[M\left(\sum_{\tau \in I_{k}} \sum_{\nu=1}^{N(k, \tau)} 2^{k s r}\left|D_{k}(f)\left(y_{\tau}^{k, \nu}\right)\right|^{r} \chi_{Q_{\tau}^{k, \nu}}\right)(x)\right]^{q / r}\right\}^{p / q} \mathrm{~d} \mu(x)\right]^{1 / p} \\
& \leqslant C\left\|\left\{\sum_{k=1}^{\infty}\left[M\left(\sum_{\tau \in I_{k}} \sum_{\nu=1}^{N(k, \tau)} 2^{k s r}\left|D_{k}(f)\left(y_{\tau}^{k, \nu}\right)\right|^{r} \chi_{Q_{\tau}^{k, \nu}}\right)\right]^{q / r}\right\}^{r / q}\right\|_{L^{p / r}(X)} \\
& \leqslant C\left\|\left\{\sum_{k=1}^{\infty} \sum_{\tau \in I_{k}} \sum_{\nu=1}^{N(k, \tau)} 2^{k s q}\left|D_{k}(f)\left(y_{\tau}^{k, \nu}\right)\right|^{q} \chi_{Q_{\tau}^{k, \nu}}\right\}^{1 / q}\right\|_{L^{p}(X)} \\
& \leqslant C\left\|\left\{\sum_{k=1}^{\infty} 2^{k s q}\left|D_{k}(f)\right|^{q}\right\}^{1 / q}\right\|_{L^{p}(X)} \\
& \leqslant C\|f\|_{F_{p q}^{s}(X)} .
\end{aligned}
$$

In the second last inequality, we have used the arbitrariness of $y_{\tau}^{k, \nu}$, and we have chosen $\epsilon \in(s, \theta)$ and $r \in(0,1]$.

We further estimate $G_{3}$ by writing

$$
\begin{aligned}
G_{3} \leqslant C \|\left\{\sum _ { l = 0 } ^ { \infty } 2 ^ { l s q } \left(2 ^ { l d } \int _ { B ( \cdot , 2 ^ { - l } ) } \left[\sum_{k=l+2}^{\infty} \sum_{\tau \in I_{k}} \sum_{\nu=1}^{N(k, \tau)} \mu\left(Q_{\tau}^{k, \nu}\right)\left|D_{k}(f)\left(y_{\tau}^{k, \nu}\right)\right|\right.\right.\right. \\
\left.\left.\left.\times\left|\tilde{D}_{k}\left(y, y_{\tau}^{k, \nu}\right)\right|\right] \mathrm{d} \mu(y)\right)^{q}\right\}^{1 / q} \|_{L^{p}(X)}
\end{aligned}
$$




$$
\begin{aligned}
& +C\left\|\left\{\sum_{l=0}^{\infty} 2^{l s q}\left[\sum_{k=l+2}^{\infty} \sum_{\tau \in I_{k}} \sum_{\nu=1}^{N(k, \tau)} \mu\left(Q_{\tau}^{k, \nu}\right)\left|D_{k}(f)\left(y_{\tau}^{k, \nu}\right)\right|\left|\tilde{D}_{k}\left(\cdot, y_{\tau}^{k, \nu}\right)\right|\right]^{q}\right\}^{1 / q}\right\|_{L^{p}(X)} \\
= & G_{3}^{1}+G_{3}^{2} .
\end{aligned}
$$

We now estimate $G_{3}^{1}$. From the size condition of $\tilde{D}_{k}$ with $\epsilon \in(0, \theta)$ and Lemma 3.10, it follows that

$$
\begin{aligned}
& G_{3}^{1} \leqslant C \|\left\{\sum _ { l = 0 } ^ { \infty } 2 ^ { l s q } \left(2 ^ { l d } \int _ { B ( \cdot , 2 ^ { - l } ) } \left[\sum_{k=l+2}^{\infty} \sum_{\tau \in I_{k}} \sum_{\nu=1}^{N(k, \tau)} \mu\left(Q_{\tau}^{k, \nu}\right)\left|D_{k}(f)\left(y_{\tau}^{k, \nu}\right)\right|\right.\right.\right. \\
& \left.\left.\left.\times \frac{2^{-k \epsilon}}{\left(2^{-k}+\rho\left(y, y_{\tau}^{k, \nu}\right)\right)^{d+\epsilon}}\right] \mathrm{d} \mu(y)\right)^{q}\right\}^{1 / q} \|_{L^{p}(X)} \\
& \leqslant C \|\left\{\sum _ { l = 0 } ^ { \infty } \left(2 ^ { l d } \int _ { B ( \cdot , 2 ^ { - l } ) } \left[\sum_{k=l+2}^{\infty} 2^{(l-k) s} \sum_{\tau \in I_{k}} \sum_{\nu=1}^{N(k, \tau)} 2^{k s}\left|D_{k}(f)\left(y_{\tau}^{k, \nu}\right)\right|\right.\right.\right. \\
& \left.\left.\left.\times \frac{1}{\left(1+2^{k} \rho\left(y, y_{\tau}^{k, \nu}\right)\right)^{d+\epsilon}}\right] \mathrm{d} \mu(y)\right)^{q}\right\}^{1 / q} \|_{L^{p}(X)} \\
& \leqslant C \|\left\{\sum _ { l = 0 } ^ { \infty } \left(2 ^ { l d } \int _ { B ( \cdot , 2 ^ { - l } ) } \left[\sum _ { k = l + 2 } ^ { \infty } 2 ^ { ( l - k ) s } M \left(\sum_{\tau \in I_{k}} \sum_{\nu=1}^{N(k, \tau)} 2^{k s}\left|D_{k}(f)\left(y_{\tau}^{k, \nu}\right)\right|\right.\right.\right.\right. \\
& \left.\left.\left.\left.\times \chi_{Q_{\tau}^{k, \nu}}\right)(y)\right] \mathrm{d} \mu(y)\right)^{q}\right\}^{1 / q} \|_{L^{p}(X)} \\
& \leqslant C\left\|\left\{\sum_{l=0}^{\infty}\left[\sum_{k=l+2}^{\infty} 2^{(l-k) s} M^{2}\left(\sum_{\tau \in I_{k}} \sum_{\nu=1}^{N(k, \tau)} 2^{k s}\left|D_{k}(f)\left(y_{\tau}^{k, \nu}\right)\right| \chi_{Q_{\tau}^{k, \nu}}\right)\right]^{q}\right\}^{1 / q}\right\|_{L^{p}(X)} .
\end{aligned}
$$

Then the Hölder inequality, the Fefferman-Stein vector-valued inequality and the fact that $y_{\tau}^{k, \nu}$ is arbitrary in $Q_{\tau}^{k, \nu}$ further yield that

$$
\begin{aligned}
G_{3}^{1} & \leqslant C \|\left\{\sum _ { l = 0 } ^ { \infty } \left(\sum_{k=l+2}^{\infty} 2^{(l-k) s}\left[M^{2}\left(\sum_{\tau \in I_{k}} \sum_{\nu=1}^{N(k, \tau)} 2^{k s}\left|D_{k}(f)\left(y_{\tau}^{k, \nu}\right)\right| \chi_{Q_{\tau}^{k, \nu}}\right)\right]^{q}\right.\right. \\
& \left.\left.\leqslant C \|\left\{\sum_{k=l+2}^{\infty} 2^{(l-k) s}\right]^{q / q^{\prime}}\right)\right\}^{1 / q} \|_{L^{p}(X)} \\
& \left.\left.\leqslant C\left\|\left\{\sum_{k=2}^{\infty}\left[M^{2}\left(\sum_{\tau=2} \sum_{\tau \in I_{k}}^{N(k, \tau)} \sum_{\nu=1}^{N(k, \tau)} 2^{k s}\left|D_{k}(f)\left(y_{\tau}^{k, \nu}\right)\right| \chi_{Q_{\tau}^{k, \nu}}\right)\right]^{q}\right\}^{1 / q}\right\|_{L^{p}(X)}(f)\left(y_{\tau}^{k, \nu}\right) \mid \chi_{Q_{\tau}^{k, \nu}}\right]^{q}\right\}^{1 / q} \|_{L^{p}(X)}
\end{aligned}
$$




$$
\begin{aligned}
& \leqslant C\left\|\left\{\sum_{k=2}^{\infty}\left[2^{k s q}\left|D_{k}(f)\right|\right]^{q}\right\}^{1 / q}\right\|_{L^{p}(X)} \\
& \leqslant C\|f\|_{F_{p q}^{s}(X)}
\end{aligned}
$$

where $M^{2}(f)=M[M(f)]$.

Finally, we estimate $G_{3}^{2}$. By the size condition of $\tilde{D}_{k}$, Hölder's inequality, Lemma 3.10 and the arbitrariness of $y_{\tau}^{k, \nu}$, we obtain

$$
\begin{aligned}
& G_{3}^{2} \\
& \leqslant C\left\|\left\{\sum_{l=0}^{\infty}\left[\sum_{k=l+2}^{\infty} 2^{(l-k) s} \sum_{\tau \in I_{k}} \sum_{\nu=1}^{N(k, \tau)} 2^{k s}\left|D_{k}(f)\left(y_{\tau}^{k, \nu}\right)\right| \frac{1}{\left(1+2^{k} \rho\left(\cdot, y_{\tau}^{k, \nu}\right)\right)^{d+\epsilon}}\right]^{q}\right\}^{1 / q}\right\|_{L^{p}(X)} \\
& \leqslant C\left\|\left\{\sum_{l=0}^{\infty} \sum_{k=l+2}^{\infty} 2^{(l-k) s}\left[M\left(\sum_{\tau \in I_{k}} \sum_{\nu=1}^{N(k, \tau)} 2^{k s r}\left|D_{k}(f)\left(y_{\tau}^{k, \nu}\right)\right|^{r} \chi_{Q_{\tau}^{k, \nu}}\right)\right]^{q / r}\right\}^{1 / q}\right\|_{L^{p}(X)} \\
& \leqslant C\left\|\left\{\sum_{k=2}^{\infty}\left[M\left(\sum_{\tau \in I_{k}} \sum_{\nu=1}^{N(k, \tau)} 2^{k s r}\left|D_{k}(f)\left(y_{\tau}^{k, \nu}\right)\right|^{r} \chi_{Q_{\tau}^{k, \nu}}\right)\right]^{q / r}\right\}^{1 / q}\right\|_{L^{p}(X)} \\
& \leqslant C\left\|\left\{\sum_{k=2}^{\infty} \sum_{\tau \in I_{k}} \sum_{\nu=1}^{N(k, \tau)} 2^{k s q}\left|D_{k}(f)\left(y_{\tau}^{k, \nu}\right)\right|^{q} \chi_{Q_{\tau}^{k, \nu}}\right\}^{1 / q}\right\| \|_{L^{p}(X)} \\
& \leqslant C\left\|\left\{\sum_{k=2}^{\infty} 2^{k s q}\left|D_{k}(f)\right|^{q}\right\}^{1 / q}\right\|_{L^{p}(X)} \\
& \leqslant C\|f\|_{F_{p q}^{s}(X),}
\end{aligned}
$$

where we chose $\epsilon \in(0, \theta)$ and $r \in(0,1]$ and we have omitted computations that are similar to $(3.22)$.

Estimates (3.19)-(3.23) now tell us that

$$
\|f\|_{L_{\mathrm{t}}(s, p, q, X)} \leqslant C\|f\|_{F_{p q}^{s}(X)} .
$$

That is, $F_{p q}^{s}(X) \subset L_{\mathrm{t}}(s, p, q, X)$, which completes the proof of Proposition 3.11.

The theorem that follows comes from Propositions 3.9 and 3.11 .

Theorem 3.12. If $0<s<\theta, 1 \leqslant p<\infty$ and $1 \leqslant q \leqslant \infty$, then

$$
L_{\mathrm{t}}(s, p, q, X)=F_{p q}^{s}(X)
$$

with equivalence of norms.

The following proposition is a simple corollary of Theorems 3.8 and 3.12 and Theorem 2 of $[\mathbf{5 3}]$ (see also Theorem 5.2 of $[\mathbf{2 3}]$ ). 
Proposition 3.13. Let $0<s_{2}<s_{1}<\theta$. Then

(i) $L\left(s_{1}, p_{1}, q, X\right) \subset L\left(s_{2}, p_{2}, q, X\right)$ for $0<q \leqslant \infty, 1 \leqslant p_{i} \leqslant \infty, i=1,2$, and $s_{1}-$ $d / p_{1}=s_{2}-d / p_{2}$

(ii) $L_{\mathrm{t}}\left(s_{1}, p_{1}, q_{1}, X\right) \subset L_{\mathrm{t}}\left(s_{2}, p_{2}, q_{2}, X\right)$ for $1 \leqslant p_{i}<\infty, 1 \leqslant q_{i} \leqslant \infty, i=1,2$, and $s_{1}-d / p_{1}=s_{2}-d / p_{2}$.

\section{Relations with other Sobolev spaces on metric spaces}

In this section, we consider the case where $X$ is a metric space with metric $\rho$. In this case, we may take $\theta$ to be 1 in Definition 1.1. The space $(X, \rho, \mu)_{d, 1}$ is then an Ahlfors $d$-regular metric space provided we further assume the Borel measure $\mu$ to be a Borel regular measure (see $[\mathbf{2 8}$, p. 25]). However, for the rest of this section it is enough to assume that $\mu$ is just a finite positive Borel measure. Let us first recall the definition of the Sobolev space of Hajłasz in [19] (see also [20]).

Definition 4.1. Let $(X, \rho, \mu)$ be a metric space and let $1 \leqslant p \leqslant \infty$. The Sobolev space $W^{1, p}(X, \rho, \mu)$ is defined by

$$
\begin{aligned}
& W^{1, p}(X, \rho, \mu)=\left\{u \in L^{p}(X) \text { : there is a set } E \subset X, \mu(E)=0,\right. \\
& \text { and a function } g \geqslant 0, g \in L^{p}(X) \text { such that } \\
& \quad|u(x)-u(y)| \leqslant \rho(x, y)(g(x)+g(y)) \text { for all } x, y \in X \backslash E\},
\end{aligned}
$$

where $g$ is called a generalized gradient of $u$. Moreover, we define

$$
\|u\|_{W^{1, p}(X, \rho, \mu)}=\|u\|_{L^{p}(X)}+\inf _{g}\|g\|_{L^{p}(X)}
$$

where the infimum is taken over all generalized gradients of the function $u$ in the definition of $W^{1, p}(X, \rho, \mu)$.

In what follows, we denote $W^{1, p}(X, \rho, \mu)$ simply by $W^{1, p}(X)$.

By Propositions 3.7 and 3.11 and a slight modification of Theorem 6.1 of [23], we easily obtain the following result.

Proposition 4.2. Let $0<s<1$ and let $X$ be an Ahlfors $d$-regular metric space. Then

(i) $W^{1, p}(X) \subset B_{p q}^{s}(X) \subset L(s, p, q, X)$ if $0<q \leqslant \infty$ and $1<p \leqslant \infty$;

(ii) $W^{1, p}(X) \subset F_{p q}^{s}(X) \subset L_{\mathrm{t}}(s, p, q, X)$ if $1<q \leqslant \infty$ and $1<p<\infty$.

The following proposition extends Proposition 4.2 to the case where $s=1$. 


\section{Proposition 4.3.}

(i) If $1 \leqslant p \leqslant \infty$, then

$$
W^{1, p}(X) \subset L(1, p, \infty, X) \subset L_{\mathrm{b}}(1, p, \infty, X) .
$$

Moreover, there is a constant $C>0$ such that for all $f \in W^{1, p}(X)$,

$$
\|f\|_{L_{\mathrm{b}}(1, p, \infty, X)} \leqslant C\|f\|_{L(1, p, \infty, X)} \leqslant C\|f\|_{W^{1, p}(X)} .
$$

(ii) If $1<p \leqslant \infty$, then $W^{1, p}(X) \subset L_{\mathrm{t}}(1, p, \infty, X)$. Moreover, there is a constant $C>0$ such that for all $f \in W^{1, p}(X)$,

$$
\|f\|_{L_{\mathrm{t}}(1, p, \infty, X)} \leqslant C\|f\|_{W^{1, p}(X)} .
$$

Proof. First we demonstrate that (i) holds. By Proposition 2.2 (ii), we need only verify the conclusions concerning the space $L(1, p, \infty, X)$. Let $f \in W^{1, p}(X)$. By Definition 3.1, there is a set $E \subset X, \mu(E)=0$ and a function $g \geqslant 0, g \in L^{p}(X)$ such that

$$
|f(x)-f(y)| \leqslant \rho(x, y)(g(x)+g(y))
$$

for all $x, y \in X \backslash E$. By (4.1) and Definition 2.1, we have

$$
\begin{aligned}
\|f\|_{L(1, p, \infty, X)}=\|f\|_{L^{p}(X)}+\sup _{\nu \in \mathbb{Z}_{+}} 2^{\nu}\left(2^{\nu d} \int_{X} \int_{B\left(x, C_{2} 2^{-\nu}\right)}|f(x)-f(y)|^{p} \mathrm{~d} \mu(y) \mathrm{d} \mu(x)\right)^{1 / p} \\
\leqslant\|f\|_{L^{p}(X)}+C \sup _{\nu \in \mathbb{Z}_{+}} 2^{\nu}\left\{2^{\nu d} \int_{X} \int_{B\left(x, C_{2} 2^{-\nu}\right)} \rho(x, y)^{p}\right. \\
\left.\quad \times\left[(g(x))^{p}+(g(y))^{p}\right] \mathrm{d} \mu(y) \mathrm{d} \mu(x)\right\}^{1 / p} \\
\leqslant C\left[\|f\|_{L^{p}(X)}+\|g\|_{L^{p}(X)}\right] .
\end{aligned}
$$

Taking the infimum on $g$ as in (4.1), we thus obtain

$$
\|f\|_{L(1, p, \infty, X)} \leqslant C\|f\|_{W^{1, p}(X)} .
$$

This proves (i).

Let us now prove (ii). Let $f \in W^{1, p}(X)$. By (4.1) and Definition 2.3, we obtain

$$
\begin{aligned}
\|f\|_{L_{\mathrm{t}}(1, p, \infty, X)} & =\|f\|_{L^{p}(X)}+\left\|\sup _{k \in \mathbb{Z}_{+}} 2^{k} 2^{k d} \int_{B\left(\cdot, C_{2} 2^{-k}\right)}|f(\cdot)-f(y)| \mathrm{d} \mu(y)\right\|_{L^{p}(X)} \\
& \leqslant\|f\|_{L^{p}(X)}+C\left\|\sup _{k \in \mathbb{Z}_{+}} 2^{k} 2^{k d} \int_{B\left(\cdot, C_{2} 2^{-k}\right)} \rho(\cdot, y)[g(\cdot)+g(y)] \mathrm{d} \mu(y)\right\|_{L^{p}(X)} \\
& \leqslant\|f\|_{L^{p}(X)}+C\|g\|_{L^{p}(X)}+C\left\|\sup _{k \in \mathbb{Z}_{+}} 2^{k d} \int_{B\left(\cdot, C_{2} 2^{-k}\right)} g(y) \mathrm{d} \mu(y)\right\|_{L^{p}(X)} \\
& \leqslant\|f\|_{L^{p}(X)}+C\|g\|_{L^{p}(X)}+C\|M(g)\|_{L^{p}(X)} \\
& \leqslant C\left[\|f\|_{L^{p}(X)}+\|g\|_{L^{p}(X)}\right]
\end{aligned}
$$


where we have used the boundedness of the Hardy-Littlewood maximal operator $M$ on $L^{p}(X)$ for $p \in(1, \infty]$ (see $[\mathbf{7}]$ and $[\mathbf{2 8}]$ ). Taking the infimum on $g$ as in (4.1), we have

$$
\|f\|_{L_{\mathrm{t}}(1, p, \infty, X)} \leqslant C\|f\|_{W^{1, p}(X)},
$$

which proves (i) and finishes the proof of Proposition 4.3.

In [34], Korevaar and Schoen define a class of Sobolev maps $f: X \rightarrow Y$, where $X$ is a Riemannian domain and $Y$ is a complete metric space. Later, Koskela and Macmanus [35] considered the Korevaar-Schoen definition in the case when the source domain is replaced by an abstract metric measure space (see also [29]). Let us recall this definition here. Let $(X, \rho, \mu)_{d, 1}$ be as in Definition 1.1 and let $f: X \rightarrow \mathbb{R}$. For any $\epsilon>0$ and $1 \leqslant p<\infty$, we define $e_{\epsilon}(x, y ; f)$ and $e_{\epsilon}(x ; f)$, respectively, by

$$
e_{\epsilon}(x, y ; f)=\frac{|f(x)-f(y)|}{\epsilon}
$$

and

$$
e_{\epsilon}^{p}(x ; f)=\frac{1}{\epsilon^{d}} \int_{B(x, \epsilon)} e_{\epsilon}(x, y ; f)^{p} \mathrm{~d} \mu(y) .
$$

Define

$$
E^{p}(f)=\sup _{B}\left(\limsup _{\epsilon \rightarrow 0} \int_{B} e_{\epsilon}^{p}(x ; f) \mathrm{d} \mu(x)\right),
$$

where the supremum is taken over all metric balls in $X$. Then $f$ is said to be in the Korevaar-Schoen Sobolev space $K S^{1, p}(X)$ if $E^{p}(f)<\infty$. Moreover, we define

$$
\|f\|_{K S^{1, p}(X)}=\|f\|_{L^{p}(X)}+\left[E^{p}(f)\right]^{1 / p} .
$$

Clearly, this definition agrees with that of Korevaar and Schoen in the case when $X$ is proper, i.e. closed balls in $X$ are compact (see [35] and Remark 5.9 in [29]).

We have the following proposition on the relationships between the Korevaar-Schoen Sobolev space and the Lipschitz-type spaces of $\S 2$.

Proposition 4.4. Let $1 \leqslant p<\infty$. Then

$$
L_{\mathrm{t}}(1, p, \infty, X) \subset L(1, p, \infty, X) \subset K S^{1, p}(X) .
$$

Furthermore, there is a constant $C>0$ such that for all $f \in L_{\mathrm{t}}(1, p, \infty, X)$,

$$
\|f\|_{K S^{1, p}(X)} \leqslant C\|f\|_{L(1, p, \infty, X)} \leqslant C\|f\|_{L_{\mathrm{t}}(1, p, \infty, X)} .
$$

Proof. The first inclusion in (4.2) can be deduced from Proposition 2.4 (iv). Hence we need only verify the second inclusion. 
Let $f \in L(1, p, \infty, X)$. Then

$$
\begin{aligned}
\|f\|_{K S^{1, p}(X)} & =\|f\|_{L^{p}(X)}+\left[E^{p}(f)\right]^{1 / p} \\
& \leqslant\|f\|_{L^{p}(X)}+\left[\limsup _{\epsilon \rightarrow 0} \int_{X} \frac{1}{\epsilon^{d}} \int_{B(x, \epsilon)} \frac{|f(x)-f(y)|^{p}}{\epsilon^{p}} \mathrm{~d} \mu(y) \mathrm{d} \mu(x)\right]^{1 / p} \\
& \leqslant\|f\|_{L^{p}(X)}+\left[\sup _{\epsilon>0} \int_{X} \frac{1}{\epsilon^{d}} \int_{B(x, \epsilon)} \frac{|f(x)-f(y)|^{p}}{\epsilon^{p}} \mathrm{~d} \mu(y) \mathrm{d} \mu(x)\right]^{1 / p} \\
& \leqslant\|f\|_{L^{p}(X)}+C\left[\sup _{\nu \in \mathbb{Z}_{+}} \int_{X} 2^{\nu p} 2^{\nu d} \int_{B\left(x, 2^{-\nu}\right)}|f(x)-f(y)|^{p} \mathrm{~d} \mu(y) \mathrm{d} \mu(x)\right]^{1 / p} \\
& \leqslant C\|f\|_{L(1, p, \infty, X)} .
\end{aligned}
$$

This means that $L(1, p, \infty, X) \subset K S^{1, p}(X)$, which completes the proof of Proposition 4.4.

Note that the relationships amongst the Sobolev space $K S^{1, p}(X)$, the Newtonian Sobolev space $N^{1, p}(X)[\mathbf{4 0}]$ and the Sobolev space $H^{1, p}(X)$ of Cheeger [4], the PoincaréSobolev spaces $P^{1, p}(X)$ and $W^{1, p}(X)$ have been established (see $[\mathbf{2 9}, \mathbf{3 5}]$ and the references therein). The following result is a simple corollary of Theorem 4.5 in $[\mathbf{3 5}]$ and Remark 5.9 in [29], and Propositions 4.3 and 4.4. See $[\mathbf{2 9}, \mathbf{3 5}]$ for the definition of the $q$-Poincaré inequality.

Corollary 4.5. Let $(X, \rho, \mu)_{d, 1}$ be a metric space as in Definition 1.1 supporting the $q$ Poncaré inequality, $1 \leqslant q<\infty$. Then for each $p \in(q, \infty)$, the spaces $W^{1, p}(X), K S^{1, p}(X)$, $H^{1, p}(X), P^{1, p}(X), L(1, p, \infty, X)$ and $L_{\mathrm{t}}(1, p, \infty, X)$ are the same space with equivalence of norms.

\section{Metric spaces with a heat kernel}

In this section, we take $\rho$ to be a metric and $\mu$ to be a non-negative Borel measure on $X$. Following [18], we say that a family $\left\{p_{t}\right\}_{t>0}$ of non-negative measurable functions $p_{t}(x, y)$ on $X \times X$ is called a heat kernel or a transition density if, for all $x, y \in X$ and $s, t>0$, the following conditions are satisfied:

$$
\begin{gathered}
p_{t}(x, y)=p_{t}(y, x) \\
\int_{X} p_{t}(x, y) \mathrm{d} \mu(y)=1 \\
p_{s+t}(x, y)=\int_{X} p_{s}(x, z) p_{t}(z, y) \mathrm{d} \mu(z)
\end{gathered}
$$

if $f \in L^{2}(X)$,

$$
\int_{X} p_{t}(x, y) f(y) \mathrm{d} \mu(y) \rightarrow f(x)
$$

in the norm of $L^{2}(X)$ as $t \rightarrow 0^{+}$. 
For example, the classical Gauss-Weierstrass function

$$
p_{t}(x, y)=\frac{1}{(4 \pi t)^{n / 2}} \exp \left(-\frac{|x-y|^{2}}{4 t}\right)
$$

is a heat kernel on $\mathbb{R}^{n}$.

Any heat kernel leads to a heat semigroup $\left\{T_{t}\right\}_{t>0}$ on $L^{2}(X)$ defined by

$$
T_{t} f(x)=\int_{X} p_{t}(x, y) f(y) \mathrm{d} \mu(y) .
$$

The above properties of $p_{t}$ tell us that $T_{t}$ is a bounded self-adjoint operator, and $\left\{T_{t}\right\}_{t>0}$ is a strongly continuous, positivity-preserving, contraction semigroup in $L^{2}(X)$.

In addition, we assume that for all $x, y \in X$ and $t \in(0, \infty)$,

$$
\frac{1}{t^{d / \beta}} \Phi_{1}\left(\frac{\rho(x, y)^{\beta}}{t}\right) \leqslant p_{t}(x, y) \leqslant \frac{1}{t^{d / \beta}} \Phi_{2}\left(\frac{\rho(x, y)^{\beta}}{t}\right),
$$

where $d$ and $\beta$ are positive constants, and $\Phi_{1}$ and $\Phi_{2}$ are monotone-decreasing positive functions on $[0, \infty)$.

Obviously, the Gauss-Weierstrass heat kernel (5.5) satisfies (5.7) with $d=n, \beta=2$ and

$$
\Phi_{1}(s)=\Phi_{2}(s)=\frac{1}{(4 \pi)^{n / 2}} \exp \left(-\frac{1}{4} s\right) .
$$

See $[\mathbf{2}]$, [11] and [18] for some other examples on fractals. In particular, for any generalized Sierpinski carpet, there exists a heat kernel satisfying the following estimate:

$$
p_{t}(x, y) \sim \frac{1}{t^{d / \beta}} \exp \left\{-C\left[\frac{\rho(x, y)^{\beta}}{t}\right]^{1 /(\beta-1)}\right\}
$$

with $2 \leqslant \beta \leqslant d+1$, which is a particular case of (5.7) (see [3] and [11]).

The following result is established by Grigoryan, Hu and Lau in [18].

Lemma 5.1. Let $(X, \rho, \mu)$ be a metric-measure space, and let $p_{t}$ be a heat kernel on $X$ satisfying (5.7) with function $\Phi_{2}$ such that

$$
\int_{C_{8}}^{\infty} s^{d / \beta} \Phi_{2}(s) \frac{\mathrm{d} s}{s}<\infty
$$

where $C_{8}>0$ is some fixed constant. Then for any ball $B(x, r)$ in $X$,

$$
\mu(B(x, r)) \sim r^{d} .
$$

Thus, $(X, \rho, \mu)$ is a special space of homogeneous type as in Definition 1.1, i.e. $(X, \rho, \mu)_{d, 1}$.

Thus, $d$ is the Hausdorff dimension of $X$. Moreover, as pointed out in [18], if the heat kernel $p_{t}$ is the transition density of a diffusion process $X_{t}$ on $X$, then $\beta$ is called the walk dimension of $X_{t}$ (see also [2]). 
We also remark that the condition (5.8) is equivalent to

$$
\int_{0}^{\infty} s^{d / \beta} \Phi_{2}(s) \frac{\mathrm{d} s}{s}<\infty
$$

since $\Phi_{2}$ is monotone decreasing.

Lemma 5.2. Let $p_{t}$ be a heat kernel on $(X, \rho, \mu)$ satisfying the upper bound in (5.7). Assume in addition that $s^{2+d / \beta} \Phi_{2}(s)$ is bounded on $\left[C_{9}, \infty\right)$ for some $C_{9}>0$. Then, for any $\sigma>\beta / 2$, the space $L(\sigma, 2, \infty, X)$ contains only constants.

Lemma 5.2 is just Theorem 4.2 in [18]. Using Lemma 5.2 and Proposition 2.2 (iv), it is easy to deduce the following strengthening of this result.

Corollary 5.3. Let $p_{t}$ be a heat kernel on $(X, \rho, \mu)$ satisfying the upper bound (5.7). Assume in addition that $s^{2+d / \beta} \Phi_{2}(s)$ is bounded on $\left[C_{9}, \infty\right)$ for some $C_{9}>0$. Then, for any $\sigma>\beta / 2$, the space $L(\sigma, 2, q, X)$ contains only constants for any $0<q \leqslant \infty$.

We now establish some Sobolev inequalities on $X$. Define by (5.6) the semigroup $\left\{T_{t}\right\}_{t>0}$ on $L^{2}(X)$, and consider the infinitesimal generator $\Delta$ defined by

$$
\Delta f=\lim _{t \rightarrow 0} \frac{T_{t} f-f}{t},
$$

where the limit is taken in the $L^{2}(X)$-norm. It is natural to refer to $\Delta$ as the Laplace operator of the heat kernel $p_{t}$. Moreover, by assumptions (5.1) and (5.2) and the Hölder inequality, it is easy to deduce that the semigroup $\left\{T_{t}\right\}_{t>0}$ is a symmetric, self-adjoint contraction which is an equicontinuous semigroup of class $\left(C_{0}\right)$ in $L^{2}(X)$ (see [54]). By the positivity of $p_{t}$, we know that $\left\{T_{t}\right\}_{t>0}$ is sub-Markovian (see [51, p. 20]). Thus, it acts on $L^{p}(X)$ for $p \in[1, \infty]$ and satisfies $\left\|T_{t}\right\|_{L^{p}(X) \rightarrow L^{p}(X)} \leqslant 1$. Moreover, it is well known that for such a semigroup, $T_{t}$ is also bounded analytic on $L^{p}(X)$ for $p \in(1, \infty)$ (see $[\mathbf{5 1}$, p. 13]). Let $\rho(\Delta)$ be the resolvent set of $\Delta$. We assume that

$$
0 \in \rho(\Delta)
$$

which is equivalent to $0 \in \rho(-\Delta)$. This can be always be achieved by multiplying the uniformly bounded semigroup $T_{t}$ by $\mathrm{e}^{-\epsilon t}$ for $\epsilon>0$. Let $R(\lambda, \Delta)=(\lambda I-\Delta)^{-1}$ be the resolvent of $\Delta$ and let $p \in(1, \infty)$. By Theorem 5.2 of $[38$, p. 61$]$, we know that if $(5.10)$ holds, the analyticity of $\left\{T_{t}\right\}_{t>0}$ is, respectively, equivalent to the following two claims:

(i) $T_{t}$ is differentiable for $t>0$ and there is a constant $C_{10}>0$ such that for $t>0$,

$$
\left\|\Delta T_{t}\right\|_{L^{p}(X) \rightarrow L^{p}(X)} \leqslant \frac{C_{10}}{t} ;
$$

or

(ii) there exist $0<\delta<\pi / 2$ and $C_{11}>0$ such that

$$
\rho(\Delta) \supset \Omega_{1}=\{\lambda:|\arg \lambda|<\pi / 2+\delta\} \cup\{0\}
$$


and

$$
\|R(\lambda, \Delta)\|_{L^{p}(X) \rightarrow L^{p}(X)} \leqslant \frac{C_{11}}{|\lambda|}
$$

for $\lambda \in \Omega_{1}$ and $\lambda \neq 0$.

Obviously, claim (ii) is equivalent to

(iii) there exist $\omega \in(0, \pi / 2)$ and $C_{12}>0$ such that

$$
\rho(-\Delta) \supset \Omega_{2}=\{\lambda: \omega<|\arg \lambda| \leqslant \pi\} \cup\{0\}
$$

and

$$
\|R(\lambda,-\Delta)\|_{L^{p}(X) \rightarrow L^{p}(X)} \leqslant \frac{C_{12}}{|\lambda|}
$$

for $\lambda \in \Omega_{2}$ and $\lambda \neq 0$.

Moreover, by using the fact that $\rho(-\Delta)$ is open and the analyticity of $R(\lambda,-\Delta)$ on $\lambda$ in each component (the maximal connected sets) of $\rho(-\Delta)$ (see Theorem 1 in [54, p. 211]), it is easy to show that (iii) is equivalent to

(iv) there exist $\omega \in(0, \pi / 2)$, a neighbourhood $V$ of 0 and $C_{13}>0$ such that

$$
\rho(-\Delta) \supset \Omega_{3}=\{\lambda: \omega<|\arg \lambda| \leqslant \pi\} \cup V
$$

and

$$
\|R(\lambda,-\Delta)\|_{L^{p}(X) \rightarrow L^{p}(X)} \leqslant \frac{C_{13}}{1+|\lambda|}
$$

for $\lambda \in \Omega_{3}$ and $\lambda \neq 0$.

Thus, $-\Delta$ is a positive operator on $L^{p}(X)$ for $p \in(1, \infty)$ in the sense of Definition 1.14.1 in [46, p. 91]. Moreover, $-\Delta$ also satisfies Assumption 6.1 in $[\mathbf{3 8}$, p. 69]. Thus, we can define the fractional powers of $-\Delta$ as in [38, pp. 69-75] (see also Section 1.15 in [46]). For $\nu \in(0,1)$, we define

$$
(-\Delta)^{-\nu} f=\int_{0}^{\infty} t^{\nu-1} T_{t} f \mathrm{~d} t, \quad(-\Delta)^{\nu} f=\left\{(-\Delta)^{-\nu}\right\}^{-1} f
$$

and $(-\Delta)^{0}=I$ (see $[\mathbf{3 8}$, pp. 70, 72]).

Let $\mathcal{F} f$ be the Fourier transform of a function $f$ on $\mathbb{R}^{n}$. In the case that $p_{t}(x, y)$ is the classical Gauss-Weierstrass function in $\mathbb{R}^{n}$ defined by $(5.5)$, then $\mathcal{F}\left\{p_{t}\right\}(\xi)=\mathrm{e}^{-t|\xi|^{2}}$ (see $[\mathbf{4 2}])$. In this case, the Laplace operator defined by (5.9) satisfies

$$
\mathcal{F}\{\Delta f\}(\xi)=\lim _{t \rightarrow 0} \frac{\mathrm{e}^{-t|\xi|^{2}}-1}{t} \mathcal{F} f(\xi)=-|\xi|^{2} \mathcal{F} f(\xi),
$$

which is just the classical Laplace operator. Moreover, since

$$
\mathcal{F}\left\{(-\Delta)^{-\nu} f\right\}(\xi)=C|\xi|^{-2 \nu} \mathcal{F} f(\xi),
$$


then

$$
(-\Delta)^{-\nu} f(x)=C \int_{\mathbb{R}}^{n} \frac{f(y)}{|x-y|^{n-2 \nu}} \mathrm{d} y
$$

(see [41]), which is just the Riesz potential. Also, since $\mathcal{F}\left\{(-\Delta)^{\nu} f\right\}(\xi)=C|\xi|^{2 \nu} \mathcal{F} f(\xi)$, $(-\Delta)^{\nu} f$ can be regarded as the derivative of fractional order of $f$. Thus, part (i) of the following theorem is the Hardy-Littlewood-Sobolev inequality (see [41]) and part (ii) is the Sobolev inequality (see [1] ). The proof of the following theorem is a slight modification of the proof of Theorem 2.4.2 in $[\mathbf{9}$, p. 75].

Theorem 5.4. Let $\left\{p_{t}\right\}_{t>0}$ be a heat kernel satisfying the upper bound estimate in (5.7) and $\Delta$ is the associated Laplace operator as in (5.9) satisfying (5.10). Let $\nu \in$ $(0,1)$ and $d>\nu \beta$. Then

(i) there exists a constant $C>0$ such that for all $f \in L^{q}(X)$,

$$
\left\|(-\Delta)^{-\nu} f\right\|_{L^{r}(X)} \leqslant C\|f\|_{L^{q}(X)},
$$

where $1<q<d / \nu \beta$ and $(1 / r)=(1 / q)-(\nu \beta / d)$;

(ii) there exists a constant $C>0$ such that for all $f \in L^{q}(X)$,

$$
\|f\|_{L^{r}(X)} \leqslant C\left\|(-\Delta)^{\nu} f\right\|_{L^{q}(X)},
$$

where $r$ and $q$ are as in (i).

Proof. Obviously, (ii) can be deduced from (i) (see also [9, pp. 75, 76]). Thus, we need only prove (i). By (5.7) and the monotonicity of $\Phi_{2}$, we have

$$
\left|T_{t} f(x)\right|=\left|\int_{X} p_{t}(x, y) f(y) \mathrm{d} \mu(y)\right| \leqslant C t^{-d / \beta}\|f\|_{L^{1}(X)} .
$$

Thus,

$$
\left\|T_{t} f\right\|_{L^{\infty}(X)} \leqslant C t^{-d / \beta}\|f\|_{L^{1}(X)} .
$$

The assumption (5.2), Hölder's inequality and (5.7) tell us that

$$
\begin{aligned}
\left|T_{t} f(x)\right| & \leqslant\left\{\int_{X} p_{t}(x, y)|f(y)|^{p} \mathrm{~d} \mu(y)\right\}^{1 / p}\left\{\int_{X} p_{t}(x, y) \mathrm{d} \mu(y)\right\}^{1 / p^{\prime}} \\
& \leqslant C t^{-d / p \beta}\|f\|_{L^{p}(X)},
\end{aligned}
$$

where $p \in[1, \infty]$ will be determined later. That is, for all $p \in[1, \infty]$,

$$
\left\|T_{t} f\right\|_{L^{\infty}(X)} \leqslant C t^{-d / p \beta}\|f\|_{L^{p}(X)} .
$$

The estimates (5.14) and (5.15) and the Riesz-Thorin interpolation theorem (see [42]) yield that for all $t>0$ and all $f \in L^{q}(X)$,

$$
\left\|T_{t} f\right\|_{L^{\infty}(X)} \leqslant C t^{-d / q \beta}\|f\|_{L^{q}(X)},
$$


where $1 \leqslant q \leqslant p$. We now write

$$
(-\Delta)^{-\nu} f=\int_{0}^{C_{14}} t^{\nu-1} T_{t} f(x) \mathrm{d} t+\int_{C_{14}}^{\infty} t^{\nu-1} T_{t} f(x) \mathrm{d} t=g+h,
$$

where $C_{14}>0$ will be determined below. By (5.16), we have

$$
\begin{aligned}
\|h\|_{L^{\infty}(X)} & \leqslant \int_{C_{14}}^{\infty} t^{\nu-1}\left\|T_{t} f\right\|_{L^{\infty}(X)} \mathrm{d} t \\
& \leqslant C\left(\int_{C_{14}}^{\infty} t^{\nu-1-(d / q \beta)} \mathrm{d} t\right)\|f\|_{L^{q}(X)} \\
& =C_{15} C_{14}^{\nu-(d / q \beta)}\|f\|_{L^{q}(X)} .
\end{aligned}
$$

For any given $\lambda>0$, we define $C_{14}$ by

$$
\frac{1}{2} \lambda=C_{15} C_{14}^{\nu-(d / q \beta)}\|f\|_{L^{q}(X)} .
$$

Note that for any $q \in[1, \infty]$, by Hölder's inequality and (5.2),

$$
\begin{aligned}
\|T g\|_{L^{q}(X)} & =\left\{\int_{X}\left|\int_{X} p_{t}(x, y) g(y) \mathrm{d} \mu(y)\right|^{q} \mathrm{~d} \mu(x)\right\}^{1 / q} \\
& =\left\{\int_{X}\left[\int_{X} p_{t}(x, y)|g(y)|^{q} \mathrm{~d} \mu(y)\right] \mathrm{d} \mu(x)\right\}^{1 / q} \\
& \leqslant\|g\|_{L^{q}(X)}
\end{aligned}
$$

from this and (5.17), we deduce

$$
\begin{aligned}
\mu\left(\left\{x \in X:\left|(-\Delta)^{-\mu} f(x)\right|>\lambda\right\}\right) & \leqslant \mu\left(\left\{x \in X:|g(x)|>\frac{1}{2} \lambda\right\}\right) \\
& \leqslant C \lambda^{-q}\|g\|_{L^{q}(X)}^{q} \\
& \leqslant C \lambda^{-q}\|f\|_{L^{q}(X)}^{q}\left(\int_{0}^{C_{14}} t^{\nu-1} \mathrm{~d} t\right)^{q} \\
& =C \lambda^{-q} C_{14}^{\nu q}\|f\|_{L^{q}(X)}^{q} \\
& =C \lambda^{-(d / \beta)((d / q \beta)-\nu)^{-1}}\|f\|_{L^{q}(X)}^{(d / \beta)((d / q \beta)-\nu)^{-1}} .
\end{aligned}
$$

Let

$$
r=\frac{d}{\beta}\left(\frac{d}{q \beta}-\nu\right)^{-1}
$$

that is,

$$
\frac{1}{r}=\frac{1}{q}-\frac{\nu \beta}{d} .
$$

Then, $(-\Delta)^{-\nu}$ is of weak type $(q, r)$, where $1 \leqslant q \leqslant p$. By choosing $p=d / \nu \beta$ and then applying the Marcinkiewicz interpolation theorem (see [42] or [41]), we obtain that $(-\Delta)^{-\nu}$ is bounded from $L^{q}(X)$ into $L^{r}(X)$, where

$$
\frac{1}{r}=\frac{1}{q}-\frac{\nu \beta}{d} \quad \text { and } \quad 1<q<\frac{d}{\nu \beta} .
$$

This finishes the proof of Theorem 5.4. 
Remark 5.5. The hypotheses of Theorem 5.4 are quite natural. Note that we only require that the heat kernel has an upper estimate as in (5.7). Upper estimates of this kind are satisfied by many heat kernels on fractals, manifolds, graphs and groups (see [3, $5,11,15,17,43,50,51])$.

In what follows, we further assume that for $0<t<t_{0}$,

$$
\sup _{s<t}\left|\frac{1}{s}\left[p_{s+t}(x, y)-p_{t}(x, y)\right]\right| \leqslant \frac{1}{t^{(d / \beta)+1}} \Phi_{3}\left(\frac{\rho(x, y)^{\beta}}{t}\right),
$$

where $t_{0}$ is some positive number and $\Phi_{3}$ is a monotone-decreasing positive function on $[0, \infty)$.

It is easy to check that the classical Gauss-Weierstrass function in (5.5) satisfies (5.18), which is also satisfied by some heat kernels on complete Riemannian manifolds (see [5] and $[\mathbf{5 0}])$.

Applying Theorem 5.4, we can obtain the following Sobolev embedding theorem (see also Theorem 4.3 of [18], Theorem 3.11 of [43] and Theorem 9.2 of [16] for some related results).

Theorem 5.6. Let $0<\nu<1$ and let $(X, \rho, \mu)$ be a metric-measure space with heat kernel satisfying (5.10) and (5.18) with

$$
\int_{C_{16}}^{\infty} t^{(d / \beta)+\nu-1} \Phi_{3}(t) \mathrm{d} t \leqslant C
$$

for some constant $C_{16}>0$. If $d>\nu \beta, 1<p<d / \nu \beta$ and

$$
\frac{1}{r}=\frac{1}{p}-\frac{\nu \beta}{d}
$$

then

$$
L_{\mathrm{b}}(\nu \beta, p, 1, X) \subset L^{r}(X) .
$$

Proof. By Theorem 5.4 (ii), we need only verify that

$$
\left\|(-\Delta)^{\nu} f\right\|_{L^{p}(X)} \leqslant C\|f\|_{L_{\mathrm{b}}(\nu \beta, p, 1, X)} .
$$

Theorem 6.8 of $[\mathbf{3 8}$, p. 72$]$ further tells us that we can write

$$
(-\Delta)^{\nu} f=(-\Delta)^{\nu-1} \circ(-\Delta) f=\int_{0}^{\infty} t^{-\nu} T_{t}(-\Delta) f \mathrm{~d} t .
$$

Moreover, Theorem 2 of $\left[\mathbf{5 4}\right.$, p. 239] tells us that $T_{t} \Delta=\Delta T_{t}$. Thus, we can write

$$
\begin{aligned}
(-\Delta)^{\nu} f(x) & =-\int_{0}^{\infty} t^{-\nu} \Delta T_{t} f(x) \mathrm{d} t \\
& =-\int_{0}^{t_{0}} t^{-\nu} \Delta T_{t} f(x) \mathrm{d} t-\int_{t_{0}}^{\infty} t^{-\nu} \Delta T_{t} f(x) \mathrm{d} t \\
& =H_{1}+H_{2} .
\end{aligned}
$$


For $0<\nu<1$, the monotonicity of $\Phi_{3}$ and (5.19) now imply that

$$
\int_{0}^{\infty} t^{(d / \beta)+\nu-1} \Phi_{3}(t) \mathrm{d} t<\infty .
$$

By (5.18), we may estimate $H_{1}$ by

$$
\begin{aligned}
\left|H_{1}\right| & =\left|\int_{0}^{t_{0}} t^{-\nu} \lim _{s \rightarrow 0} \frac{T_{s} T_{t} f(x)-T_{t} f(x)}{s} \mathrm{~d} t\right| \\
& =\left|\int_{0}^{t_{0}} t^{-\nu} \lim _{\substack{s \rightarrow 0 \\
s<t}} \int_{X} \frac{1}{s}\left[p_{s+t}(x, y)-p_{t}(x, y)\right][f(y)-f(x)] \mathrm{d} \mu(y) \mathrm{d} t\right| \\
& \leqslant \int_{0}^{t_{0}} t^{-\nu}\left[\int_{X} \frac{1}{t^{(d / \beta)+1}} \Phi_{3}\left(\frac{\rho(x, y)^{\beta}}{t}\right)|f(y)-f(x)| \mathrm{d} \mu(y)\right] \mathrm{d} t \\
& \leqslant \int_{0}^{t_{0}} t^{-\nu}\left[\int_{X \backslash B(x, 1)} \cdots \mathrm{d} \mu(y)\right] \mathrm{d} t+\int_{0}^{t_{0}} t^{-\nu}\left[\int_{B(x, 1)} \cdots \mathrm{d} \mu(y)\right] \mathrm{d} t \\
& =H_{1}^{1}+H_{1}^{2} .
\end{aligned}
$$

We have, by (5.21),

$$
\begin{aligned}
H_{1}^{1} & \leqslant \int_{X \backslash B(x, 1)}\left[\int_{0}^{t_{0}} \frac{1}{t^{(d / \beta)+1+\nu}} \Phi_{3}\left(\frac{\rho(x, y)^{\beta}}{t}\right) \mathrm{d} t\right]|f(y)-f(x)| \mathrm{d} \mu(y) \\
& \leqslant C\left[\int_{0}^{\infty} t^{(d / \beta)+\mu-1} \Phi_{3}(t) \mathrm{d} t\right] \int_{X \backslash B(x, 1)} \frac{1}{\rho(x, y)^{d+\nu \beta}}|f(y)-f(x)| \mathrm{d} \mu(y) \\
& \leqslant C \int_{X \backslash B(x, 1)} \frac{1}{\rho(x, y)^{d+\nu \beta}}|f(y)-f(x)| \mathrm{d} \mu(y) \\
& \leqslant C|f(x)|+C \int_{X \backslash B(x, 1)} \frac{1}{\rho(x, y)^{d+\nu \beta}}|f(y)| \mathrm{d} \mu(y) .
\end{aligned}
$$

Since $d+\nu \beta>d$, we may use this, together with Hölder's inequality, to deduce

$$
\begin{aligned}
\left\|H_{1}^{1}\right\|_{L^{p}(X)} & \leqslant C\|f\|_{L^{p}(X)}+C\left\{\int_{X}\left[\int_{X \backslash B(x, 1)} \frac{1}{\rho(x, y)^{d+\nu \beta}}|f(y)|^{p} \mathrm{~d} \mu(y)\right]\right. \\
& \left.\times\left[\int_{X \backslash B(x, 1)} \frac{1}{\rho(x, y)^{d+\nu \beta}} \mathrm{d} \mu(y)\right] \mathrm{d} \mu(x)\right\}^{1 / p} \\
& \leqslant C\|f\|_{L^{p}(X)} \\
& \leqslant C\|f\|_{L_{\mathrm{b}}(\nu \beta, p, 1, X)} .
\end{aligned}
$$

Again using (5.21), we estimate $H_{1}^{2}$ by decomposing it as

$$
\begin{aligned}
H_{1}^{2} & \leqslant \sum_{k=1}^{\infty} \int_{0}^{t_{0}} \int_{B\left(x, 2^{-(k-1)}\right) \backslash B\left(x, 2^{-k}\right)} \frac{1}{t^{(d / \beta)+1+\nu}} \Phi_{3}\left(\frac{\rho(x, y)^{\beta}}{t}\right)|f(y)-f(x)| \mathrm{d} \mu(y) \mathrm{d} t \\
& \leqslant \sum_{k=1}^{\infty} \int_{0}^{t_{0}} \frac{1}{t^{(d / \beta)+1+\nu}} \Phi_{3}\left(\frac{2^{-k \beta}}{t}\right) \mathrm{d} t \int_{B\left(x, 2^{-(k-1)}\right)}|f(y)-f(x)| \mathrm{d} \mu(y)
\end{aligned}
$$




$$
\begin{aligned}
& \leqslant C\left[\int_{0}^{\infty} t^{(d / \beta)+\nu-1} \Phi_{3}(t) \mathrm{d} t\right] \sum_{k=1}^{\infty} 2^{k \beta \nu} 2^{k d} \int_{B\left(x, 2^{-(k-1)}\right)}|f(y)-f(x)| \mathrm{d} \mu(y) \\
& \leqslant C \sum_{k=1}^{\infty} 2^{k \beta \nu} 2^{k d} \int_{B\left(x, 2^{-(k-1)}\right)}|f(y)-f(x)| \mathrm{d} \mu(y) .
\end{aligned}
$$

From this it follows that

$$
\begin{aligned}
\left\|H_{1}^{2}\right\|_{L^{p}(X)} & \leqslant C \sum_{k=1}^{\infty} 2^{k \beta \nu}\left\{\int_{X}\left[2^{k d} \int_{B\left(x, 2^{-(k-1)}\right)}|f(y)-f(x)| \mathrm{d} \mu(y)\right]^{p} \mathrm{~d} \mu(x)\right\}^{1 / p} \\
& \leqslant C\|f\|_{L_{\mathrm{b}}(\nu \beta, p, 1, X)} .
\end{aligned}
$$

Estimates (5.22) and (5.23) tell us that

$$
\left\|H_{1}\right\|_{L^{p}(X)} \leqslant C\|f\|_{L_{\mathrm{b}}(\nu \beta, p, 1, X)} .
$$

We now estimate $H_{2}$. From (5.11) and the Minkowski inequality for integrals, it follows that

$$
\left\|H_{2}\right\|_{L^{p}(X)} \leqslant \int_{t_{0}}^{\infty} t^{-\nu}\left\|\Delta T_{t} f\right\|_{L^{p}(X)} \mathrm{d} t \leqslant C \int_{t_{0}}^{\infty} t^{-\nu-1} \mathrm{~d} t\|f\|_{L^{p}(X)} \leqslant C\|f\|_{L^{p}(X)} .
$$

This inequality, combined with (5.24), tells us that (5.20) is valid.

This completes the proof of Theorem 5.6.

Remark 5.7. Let $s=\nu \beta$. Then the condition $1<p<d / s$ implies that $s<d / p$. Thus, Theorem 5.6 extends Corollary 2.6 in the current setting.

Acknowledgements. D.Y. was supported by the NNSF (no. 10271015) and the RFDP (no. 20020027004) of China, and he thanks Professor Hans Triebel, Professor Robert S. Strichartz, Professor Anthony H. Dooley and Dr Jiaxin Hu for some helpful discussions on this subject. Y.L. also thanks Dr Lifeng Xi and Professor J. Kigami for several useful comments on this paper.

\section{References}

1. R. A. Adams, Sobolev spaces (Academic, 1975).

2. M. T. BARLOW, Diffusions on fractals, in Lectures on probability theory and statistics (Saint-Flour, 1995) (ed. P. Bernard), Lecture Notes in Mathematics, vol. 1690, pp. 1-121 (Springer, 1998).

3. M. T. BARLOW AND R. F. BASS, Brownian motion and harmonic analysis on Sierpinski carpets, Can. J. Math. 51 (1999), 673-744.

4. J. Cheeger, Differentiability of Lipschitz functions on metric measure spaces, Geom. Funct. Analysis 9 (1999), 428-517.

5. S. Y. Cheng, P. Li AND S.-T. YAU, On the upper estimate of the heat kernel of a complete Riemannian manifold, Am. J. Math. 103 (1981), 1021-1063.

6. M. Christ, A $T(b)$ theorem with remarks on analytic capacity and the Cauchy integral, Colloq. Math. LX/LXI (1990), 601-628. 
7. R. R. COIFMAN AND G. WEISS, Analyse harmonique non-commutative sur certains espaces homogènes, Lecture Notes in Mathematics, vol. 242 (Springer, 1971).

8. G. David, J. L. Journé And S. Semmes, Opérateurs de Calderón-Zygmund, fonctions para-accrétives et interpolation, Rev. Mat. Iber. 1 (1985), 1-56.

9. E. B. DAviEs, Heat kernels and spectral theory (Cambridge University Press, 1989).

10. C. Fefferman And E. M. Stein, Some maximal inequalities, Am. J. Math. 93 (1971), 107-116.

11. P. J. Fitzsimmons, B. M. Hambly and T. Kumagai, Transition density estimates for Brownian motion on affine nested fractals, Commun. Math. Phys. 165 (1994), 595-620.

12. M. FrAZIER AND B. JAWERTH, A discrete transform and decompositions of distribution spaces, J. Funct. Analysis 93 (1990), 34-170.

13. A. E. Gatтo, Product rule and chain rule estimates for fractional derivatives on spaces that satisfy the doubling condition, J. Funct. Analysis 188 (2002), 27-37.

14. A. E. Gatto, C. Segovia And S. VÁGi, On fractional differentiation and integration on spaces of homogeneous type, Rev. Mat. Iber. 12 (1996), 111-145.

15. A. Grigor'yan, Estimates of heat kernels on Riemannian manifolds, in Spectral theory and geometry (Edinburgh, 1998) (ed. B. Davies and Y. Safarov), London Mathematical Society Lecture Note Series, vol. 273, pp. 140-225 (Cambridge University Press, 1999).

16. A. GRIGOR'YAn, Heat kernels and function theory on metric measure spaces, in Heat Kernels and Analysis on Manifolds, Graphs, and Metric Spaces, Paris, 2002 (ed. P. Auscher, T. Coulhon and A. Grigor'yan), pp. 143-172, Contemporary Mathematics, vol. 338 (American Mathematical Society, Providence, RI, 2003).

17. A. GRigor'yan And A. Telcs, Sub-Gaussian estimates of heat kernels on infinite graphs, Duke Math. J. 109 (2001), 451-510.

18. A. GRigor'yan, J. Hu AND K. LAU, Heat kernels on metric-measure spaces and an application to semi-linear elliptic equations, Trans. Am. Math. Soc. 355 (2003), 20652095.

19. P. HajŁasz, Sobolev spaces on an arbitrary metric space, Potent. Analysis 5 (1996), 403-415.

20. P. HajŁasz and P. Koskela, Sobolev met Poincaré, pp. 1-101, Memoirs of the American Mathematical Society, vol. 145 (2000).

21. Y. HAN, Inhomogeneous Calderón reproducing formula on spaces of homogeneous type, J. Geom. Analysis 7 (1997), 259-284.

22. Y. HAN AND E. T. SAWYER, Littlewood-Paley theory on spaces of homogeneous type and classical function spaces, pp. 1-126, Memoirs of the American Mathematical Society, vol. 110 (1994).

23. Y. HAN AND D. YANG, New characterizations and applications of inhomogeneous Besov and Triebel-Lizorkin spaces on homogeneous type spaces and fractals, Dissertationes Math. (Rozprawy Mat.) 403 (2002), 1-102.

24. Y. HAN AND D. YANG, Some new spaces of Besov and Triebel-Lizorkin type on homogeneous spaces, Studia Math. 156 (2003), 67-97.

25. Y. HAN, S. LU AND D. YAnG, Inhomogeneous Besov and Triebel-Lizorkin spaces on spaces of homogeneous type, Approx. Theory Applicat. 15(3) (1999), 37-65.

26. Y. HAN, S. LU AND D. YANG, Inhomogeneous Triebel-Lizorkin spaces on spaces of homogeneous type, Math. Sci. Res. Hot-Line 3(9) (1999), 1-29.

27. Y. HAN, S. LU AND D. YANG, Inhomogeneous discrete Calderón reproducing formulas for spaces of homogeneous type, J. Fourier Analysis Applic. 7 (2001), 571-600.

28. J. HEINONEN, Lectures on analysis on metric spaces (Springer, 2001).

29. J. Heinonen, P. Koskela, N. Shanmugalingam and J. T. Tyson, Sobolev classes of Banach space-valued functions and quasiconformal mappings, J. Analysis Math. 85 (2001), 87-139. 
30. A. Jonsson, Brownian motion on fractals and function spaces, Math. Z. 222 (1996), 495-504.

31. A. Jonsson And H. WAllin, Function spaces on subsets of $\mathbb{R}^{n}$, Mathematical Reports, vol. 2 (Harwood Academic, New York, 1984).

32. A. Jonsson AND H. WALLin, Boundary value problems and Brownian motion on fractals, Chaos Solitons Fractals 8 (1997), 191-205.

33. J. Kigami, Analysis on fractals, Cambridge Tracts in Mathematics, vol. 143 (Cambridge University Press, 2001).

34. N. J. KorevaAR And R. M. Schoen, Sobolev spaces and harmonic maps for metric spaces targets, Commun. Analysis Geom. 1 (1993), 561-659.

35. P. Koskela And P. MacManus, Quasiconformal mappings and Sobolev spaces, Studia Math. 131 (1998), 1-17.

36. R. A. Macías And C. Segovia, Lipschitz functions on spaces of homogeneous type, $A d v$. Math. 33 (1979), 257-270.

37. P. Mattila, Geometry of sets and measures in Euclidean spaces (Cambridge University Press, 1995).

38. A. PAZY, Semigroups of linear operators and applications to partial differential equations (Springer, 1983).

39. S. Semmes, Happy fractals and some aspects of analysis on metric spaces, Publ. Mat. 47 (2003), 261-309.

40. N. Shanmugalingam, Newtonian spaces: an extension of Sobolev spaces to metric measure spaces, Rev. Mat. Iber. 16 (2000), 243-279.

41. E. M. STEIN, Singular integrals and differentiability properties of functions (Princeton University Press, 1970).

42. E. M. STEIN AND G. WeISS, Introduction to Fourier analysis on Euclidean spaces (Princeton University Press, 1971).

43. R. S. Strichartz, Function spaces on fractals, J. Funct. Analysis 198 (2003), 43-83.

44. H. TRIEBEL, Theory of function spaces (Birkhäuser, 1983).

45. H. TRIEBEL, Theory of function spaces, vol. II (Birkhäuser, 1992).

46. H. TRIEBEL, Interpolation theory, function spaces, differential operators, 2nd edn (Johann Ambrosius Barth, Heidelberg, 1995).

47. H. TRIEBEL, Fractals and spectra (Birkhäuser, 1997).

48. H. TRIEBEL, The structure of functions (Birkhäuser, 2001).

49. H. TRIEBEL, Function spaces in Lipschitz domains and on Lipschitz manifolds, Characteristic functions as pointwise multipliers, Rev. Mat. Complut. 15 (2002), 475-524.

50. N. Th. Varopoulos, Hardy-Littlewood theory for semigroups, J. Funct. Analysis 63 (1985), 240-260.

51. N. Th. Varopoulos, L. Saloff-Coste and T. Coulhon, Analysis and geometry on groups (Cambridge University Press, 1992).

52. D. YANG, Besov spaces on spaces of homogeneous type and fractals, Studia Math. 156 (2003), 15-30.

53. D. YANG, Embedding theorems of Besov and Triebel-Lizorkin spaces on spaces of homogeneous type, Sci. China A 46 (2003), 187-199.

54. K. YosidA, Functional analysis, 6th edn (Springer, 1980). 